\title{
Incidence and survival of remnant disks around main-sequence stars ${ }^{\star, \star \star}$
}

\author{
H. J. Habing ${ }^{1}$, C. Dominik ${ }^{1}$, M. Jourdain de Muizon ${ }^{2,3}$, R. J. Laureijs ${ }^{4}$, M. F. Kessler ${ }^{4}$, K. Leech ${ }^{4}$, \\ L. Metcalfe ${ }^{4}$, A. Salama ${ }^{4}$, R. Siebenmorgen ${ }^{4}$, N. Trams ${ }^{4}$, and P. Bouchet ${ }^{5}$ \\ 1 Sterrewacht, Leiden, PO Box 9513, 2300 RA Leiden, The Netherlands \\ 2 DESPA, Observatoire de Paris, 92190 Meudon, France \\ 3 LAEFF-INTA, ESA Vilspa, PO Box 50727, 28080 Madrid, Spain \\ ${ }^{4}$ ISO Data Center, Astrophysics Division of ESA, Vilspa, PO Box 50727, 28080 Madrid, Spain \\ 5 Cerro Tololo Inter-American Observatory, NOAO, Casilla 603, La Serena, Chile 1353
}

Received 8 August 2000 / Accepted 26 October 2000

\begin{abstract}
We present photometric ISO 60 and 170 um measurements, complemented by some IRAS data at $60 \mu \mathrm{m}$, of a sample of 84 nearby main-sequence stars of spectral class A, F, G and $\mathrm{K}$ in order to determine the incidence of dust disks around such main-sequence stars. Fifty stars were detected at $60 \mu \mathrm{m} ; 36$ of these emit a flux expected from their photosphere while 14 emit significantly more. The excess emission we attribute to a circumstellar disk like the ones around Vega and $\beta$ Pictoris. Thirty four stars were not detected at all; the expected photospheric flux, however, is so close to the detection limit that the stars cannot have an excess stronger than the photospheric flux density at $60 \mu \mathrm{m}$. Of the stars younger than $400 \mathrm{Myr}$ one in two has a disk; for the older stars this is true for only one in ten. We conclude that most stars arrive on the main sequence surrounded by a disk; this disk then decays in about 400 Myr. Because (i) the dust particles disappear and must be replenished on a much shorter time scale and (ii) the collision of planetesimals is a good source of new dust, we suggest that the rapid decay of the disks is caused by the destruction and escape of planetesimals. We suggest that the dissipation of the disk is related to the heavy bombardment phase in our Solar System. Whether all stars arrive on the main sequence surrounded by a disk cannot be established: some very young stars do not have a disk. And not all stars destroy their disk in a similar way: some stars as old as the Sun still have significant disks.
\end{abstract}

Key words. stars: planetary systems - infrared: stars

\section{Introduction}

In 1983, while using standard stars to calibrate the IRAS photometry, Aumann et al. (1984) discovered that Vega ( $\alpha$ Lyr), one of the best calibrated and most used photometric standards in the visual wavelength range, emits much more energy at mid- and far-infrared wavelengths than its photosphere produces. Because the star is not reddened Aumann et al. proposed that the excess IR radiation is emitted by small, interplanetary-dust particles in a disk rather than in a spherical envelope. This proposal was confirmed by Smith \& Terrile (1984) who detected

Send offprint requests to: H. J. Habing,

e-mail: habing@strw. leidenuniv.nl

* Based on observations with ISO, an ESA project with instruments funded by ESA Member States (especially the PI countries: France, Germany, The Netherlands and the UK) and with the participation of ISAS and NASA.

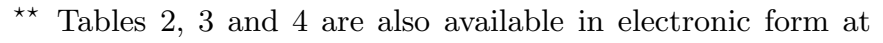
the CDS via anonymous ftp

cdsarc.u-strasbg.fr (130.79.128.5) or via

http://cdsweb.u-strasbg.fr/cgi-bin/qcat?J/A+A/365/545 a flat source of scattered light around $\beta$ Pic, one of the other Vega-like stars detected in the IRAS data (Gillett 1986), and the one with the strongest excess. The disk around Vega and other main-sequence stars is the remnant of a much stronger disk built up during the formation of the stars. Aumann et al. (1984) pointed out that such disks have a lifetime much shorter than the stellar age and therefore need to be rebuilt continuously; collisions between asteroids are a probable source of new dust (Weissman 1984). Except for the somewhat exceptional case of $\beta$ Pic (Hobbs et al. 1985) and in spite of several deep searches no trace of any gas has ever been found in the disks around main-sequence stars; see e.g. Liseau (1999).

Since 1984 the search for and the study of remnant disks has made substantial progress by the discovery of numerous "fatter" disks around pre-main sequence stars that contain dust and gas; for overviews see Beckwith \& Sargent (1996), Sargent \& Welch (1993), van Dishoeck \& Blake (1998). The IRAS data base also contains detections of Vega-like disks around red giant 
stars that have developed from A and F-type mainsequence stars (Plets et al. 1997).

The discovery of Aumann et al. has prompted deeper searches in the IRAS data base with different strategies (Aumann 1985; Walker \& Wolstencroft 1988; Mannings \& Barlow 1998). For a review see Backman \& Paresce (1993). Recently Plets \& Vynckier (1999) have discussed these earlier results and concluded that a significant excess at $60 \mu \mathrm{m}$ is found in $13 \pm 10 \%$ of all main sequence stars with spectral type A, F, G and K. Unfortunately all these studies based on IRAS data only were affected by severe selection effects and did not answer important questions such as: will a star loose its disk when it grows older? On what time-scale? Does the presence of planets depend on the stellar main-sequence mass? Do multiple stars have disks more, or less frequently? Do stars that formed in clusters have disks less often? With such questions unanswered we clearly do not understand the systematics of the formation of solar systems.

Here we present results of a continuation with ISO (Kessler et al. 1996) of the succesful search of IRAS. Our aim has been to obtain a better defined sample of stars. The major step forward in this paper is not in the detection of more remnant disks, but in reliable information about the presence or absence of a disk. Earlier reports on results from our program have been given in Habing et al. (1996), Dominik et al. (1998), Jourdain de Muizon et al. (1999) and Habing et al. (1999).

\section{Selecting and preparing the sample}

Stars were selected so that their photospheric flux was within our sensitivity limit. Any excess would then appear immediately. We also wanted to make certain that any excess flux should be attributed to a circumstellar disk and not to some other property of the star, such as circumstellar matter ejected during the stellar evolution or to the presence of a red companion.

In selecting our stars we used the following criteria:

- We selected main-sequence stars with an expected photospheric flux at $60 \mu \mathrm{m}$ larger than $30 \mathrm{mJy}$. We started from a list of infrared flux densities calculated by Johnson \& Wright (1983) for 2000 stars contained in a catalogue of stars within 25 pc by Woolley et al. (1970). The limit of $30 \mathrm{mJy}$ was based on the sensitivity of ISOPHOT (Lemke et al. 1996) as announced before launch by Klaas et al. (1994);

- We removed all stars with peculiarities in their spectra for which an accurate infrared flux density could not be predicted. This made us eliminate all $\mathrm{O}$ and $\mathrm{B}$ stars (emission lines, free-free IR excess) and all M stars (molecular spectra not well understood);

- We also removed all spectroscopic double stars. Visual double stars were rejected when the companion lies within 1 arcmin and its $V$-magnitude differs by less than 5 magnitudes; any remaining companion will contribute less than $10 \%$ of the $60 \mu \mathrm{m}$ flux as one may show through Eq. (1);
Table 1. Apparent magnitude and distance from the Sun (in parsec) of main-sequence stars with a $60 \mu \mathrm{m}$ flux density of $30 \mathrm{mJy}$

\begin{tabular}{|c|cccccccc|}
\hline Sp. Type & A0 & A5 & F0 & F5 & G0 & G5 & K0 & K5 \\
\hline$V(\mathrm{mag})$ & 4.0 & 4.4 & 4.8 & 5.2 & 5.7 & 6.0 & 6.8 & 7.4 \\
$d(\mathrm{pc})$ & 45 & 31 & 25 & 19 & 15 & 13 & 10 & 7.5 \\
\hline
\end{tabular}

- We also excluded variable stars; the variability of all stars has been checked a posteriori using the photometry in the band at $0.6 \mu \mathrm{m}$ given in the Hipparcos catalogue (Perryman et al. 1997); in all cases the stellar magnitude is constant within 0.07 magnitude.

To illustrate what stars are bright enough to be included we use an equation that gives the stellar colour, $(V-[60 \mu \mathrm{m}])$, as a function of $(B-V)$. The equation has been derived empirically from IRAS data by Waters et al. (1987); we use a slightly different version given by H. Plets (private communication):

$$
\begin{aligned}
V-[60 \mu \mathrm{m}]= & 0.01+2.99(B-V) \\
& -1.06(B-V)^{2}+0.47(B-V)^{3} .
\end{aligned}
$$

The zero point in this equation has a formal error of 0.01 . Intrinsic, reddening-free $(B-V)$ values must be used, but all our stars are nearby and we assume that the measured values are reddening-free. A posteriori we checked that we may safely ignore the reddening produced by the disks that we detected; only in the case of $\beta$ Pic is a small effect expected. Adopting a flux density of $1.19 \mathrm{Jy}$ for $[60 \mu \mathrm{m}]=0$ we find apparent-magnitude limits and distance limits of suitable main-sequence stars as summarized in Table 1. The distance limit varies strongly with spectral type.

Table 2 contains basic data on all stars from the sample for which we present ISO data. Columns 1 and 2 contain the number of the star in the HD and in the Hipparcos Catalogue (Perryman et al. 1997) and Col. 3 the name. $V$ and $B-V$ have been taken from the Geneva photometric catalogue (Kunzli et al. 1997). Columns 6 and 7 contain the distance and the spectral type as given in the Hipparcos Catalog (Perryman et al. 1997). The age given in Col. 8 is from Lachaume et al. (1999), where errors in the age determinations are discussed. The effective temperature in Col. 11 has been derived by fitting Kurucz' model atmospheres to the Geneva photometry; we will need this temperature to calculate the dust mass from the flux-density excess at $60 \mu \mathrm{m}$.

\section{Measurements, data reduction, checks}

\subsection{Measurements}

Pre-launch recommendations made us start with chopped measurements (observing mode PHT03; see Laureijs et al. $2000)$ at $60,90,135$ and $170 \mu \mathrm{m}$. After a few months of operation of the satellite it appeared that at 60 and $90 \mu \mathrm{m}$ the on-off signal was strongly distorted by transients in 
Table 2. The stars of the sample

\begin{tabular}{|c|c|c|c|c|c|c|c|c|}
\hline $\begin{array}{l}\text { HD } \\
\text { (1) }\end{array}$ & $\begin{array}{r}\text { HIP } \\
(2) \\
\end{array}$ & $\begin{array}{l}\text { Name } \\
(3)\end{array}$ & $\begin{array}{r}V \\
\operatorname{mag} \\
(4)\end{array}$ & $\begin{array}{r}B-V \\
\text { mag } \\
(5)\end{array}$ & $\begin{array}{r}d \\
\text { pc } \\
(6) \\
\end{array}$ & $\begin{array}{l}\text { Spect. } \\
(7)\end{array}$ & $\begin{array}{r}\text { age } \\
\text { Gyrs } \\
(8)\end{array}$ & $\begin{array}{r}T_{\text {eff }} \\
\mathrm{K} \\
(9)\end{array}$ \\
\hline 693 & 910 & 6 Cet & 4.89 & 0.49 & 18.9 & F5V & 5.13 & 6210 \\
\hline 1581 & 1599 & $\zeta$ Tuc & 4.23 & 0.58 & 8.6 & F9V & 6.46 & 5990 \\
\hline 2151 & 2021 & $\beta$ Нyi & 2.82 & 0.62 & 7.5 & G2IV & 5.37 & 5850 \\
\hline 4628 & 3765 & & 5.74 & 0.89 & 7.5 & $\mathrm{~K} 2 \mathrm{~V}$ & 7.94 & 5050 \\
\hline 4813 & 3909 & $\phi^{2}$ Cet & 5.17 & 0.51 & 15.5 & F7IV-V & 1.38 & 6250 \\
\hline 7570 & 5862 & $\nu$ Phe & 4.97 & 0.57 & 15.1 & F8V & 3.16 & 6080 \\
\hline 9826 & 7513 & 50 And & 4.10 & 0.54 & 13.5 & F8V & 2.88 & 6210 \\
\hline 10700 & 8102 & $\tau$ Cet & 3.49 & 0.73 & 3.6 & G8V & 7.24 & 5480 \\
\hline 10780 & 8362 & & 5.63 & 0.80 & 10.0 & KOV & 2.82 & 5420 \\
\hline 12311 & 9236 & $\alpha \mathrm{Hyi}$ & 2.86 & 0.29 & 21.9 & FoV & 0.81 & 7080 \\
\hline 13445 & 10138 & & 6.12 & 0.81 & 10.9 & KOV & 5.37 & 5400 \\
\hline 14412 & 10798 & & 6.33 & 0.72 & 12.7 & G8V & 7.24 & 5420 \\
\hline 14802 & 11072 & $\kappa$ For & 5.19 & 0.61 & 21.9 & $\mathrm{G} 2 \mathrm{~V}$ & 5.37 & 5850 \\
\hline 15008 & 11001 & $\delta$ Hyi & 4.08 & 0.03 & 41.5 & A3V & 0.45 & 8920 \\
\hline 17051 & 12653 & $\iota$ Hor & 5.40 & 0.56 & 17.2 & G3IV & 3.09 & 6080 \\
\hline 17925 & 13402 & & 6.05 & 0.86 & 10.4 & $\mathrm{~K} 1 \mathrm{~V}$ & 0.08 & 5000 \\
\hline 19373 & 14632 & $\iota$ Per & 4.05 & 0.60 & 10.5 & GoV & 3.39 & 6040 \\
\hline 20630 & 15457 & $\kappa^{1}$ Cet & 4.84 & 0.68 & 9.2 & G5Vv & 0.30 & 5750 \\
\hline 20766 & 15330 & $\zeta^{1}$ Ret & 5.53 & 0.64 & 12.1 & $\mathrm{G} 2 \mathrm{~V}$ & 4.79 & 5750 \\
\hline 20807 & 15371 & $\zeta^{2}$ Ret & 5.24 & 0.60 & 12.1 & G1V & 7.24 & 5890 \\
\hline 22001 & 16245 & $\kappa$ Ret & 4.71 & 0.41 & 21.4 & F5IV-V & 2.04 & 6620 \\
\hline 22049 & 16537 & $\epsilon$ Eri & 3.72 & 0.88 & 3.2 & $\mathrm{~K} 2 \mathrm{~V}$ & 0.33 & 5000 \\
\hline 22484 & 16852 & $10 \mathrm{Tau}$ & 4.29 & 0.58 & 13.7 & F9V & 5.25 & 5980 \\
\hline 23249 & 17378 & $\delta$ Eri & 3.52 & 0.92 & 9.0 & $\mathrm{~K} 2 \mathrm{~V}$ & 7.59 & 5000 \\
\hline 26965 & 19849 & $o^{2}$ Eri & 4.43 & 0.82 & 5.0 & $\mathrm{~K} 1 \mathrm{~V}$ & 7.24 & 5100 \\
\hline 30495 & 22263 & 58 Eri & 5.49 & 0.63 & 13.3 & G3V & 0.21 & 5820 \\
\hline 33262 & 23693 & $\zeta$ Dor & 4.71 & 0.53 & 11.7 & F7V & 2.95 & 6160 \\
\hline 34411 & 24813 & $\lambda$ Aur & 4.69 & 0.63 & 12.7 & G0V & 6.76 & 5890 \\
\hline 37394 & 26779 & & 6.21 & 0.84 & 12.2 & $\mathrm{~K} 1 \mathrm{~V}$ & 0.34 & 5100 \\
\hline 38392 & & & 6.15 & 0.94 & 9.0 & $\mathrm{~K} 2 \mathrm{~V}$ & 0.87 & 4950 \\
\hline 38393 & 27072 & $\gamma$ Lep & 3.59 & 0.48 & 9.0 & F7V & 1.66 & 6400 \\
\hline 38678 & 27288 & $\zeta$ Lep & 3.55 & 0.10 & 21.5 & A2Vann & 0.37 & 8550 \\
\hline 39060 & 27321 & $\beta \mathrm{Pic}$ & 3.85 & 0.17 & 19.3 & $\mathrm{~A} 3 \mathrm{~V}$ & 0.28 & 8040 \\
\hline 43834 & 29271 & $\alpha$ Men & 5.08 & 0.71 & 10.2 & G5V & 7.24 & 5630 \\
\hline 48915 & 32349 & $\alpha \mathrm{CMa}$ & -1.44 & 0.01 & 2.6 & $\mathrm{~A} 0 \mathrm{~m}$ & & 9920 \\
\hline 50281 & 32984 & & 6.58 & 1.07 & 8.7 & K3V & 2.63 & 5000 \\
\hline 61421 & 37279 & $\alpha \mathrm{CMi}$ & 0.40 & 0.43 & 3.5 & F5IV-V & 1.70 & 6700 \\
\hline 74956 & 42913 & $\delta \mathrm{Vel}$ & 1.93 & 0.04 & 25.0 & $\mathrm{~A} 1 \mathrm{~V}$ & 0.35 & 9200 \\
\hline 75732 & 43587 & $\rho^{1} \mathrm{Cnc}$ & 5.96 & 0.87 & 12.5 & G8V & 5.01 & 5300 \\
\hline 80007 & 45238 & $\beta$ Car & 1.67 & 0.07 & 34.1 & A2IV & & 8600 \\
\hline 95418 & 53910 & $\beta \mathrm{UMa}$ & 2.34 & 0.03 & 24.4 & $\mathrm{~A} 1 \mathrm{~V}$ & 0.36 & 9530 \\
\hline 102647 & 57632 & $\beta$ Leo & 2.14 & 0.09 & 11.1 & A3Vvar & 0.24 & 8580 \\
\hline 102870 & 57757 & $\beta$ Vir & 3.59 & 0.52 & 10.9 & F8V & 2.63 & 6180 \\
\hline 103287 & 58001 & $\gamma \mathrm{UMa}$ & 2.41 & 0.04 & 25.7 & A0V SB & 0.38 & 9440 \\
\hline 106591 & 59774 & $\delta \mathrm{UMa}$ & 3.32 & 0.08 & 25.0 & A3Vvar & 0.48 & 8630 \\
\hline 110833 & 62145 & & 7.01 & 0.94 & 15.1 & K3V & 12.60 & 5000 \\
\hline 112185 & 62956 & $\epsilon \mathrm{UMa}$ & 1.76 & -0.02 & 24.8 & A0p & 0.30 & 9780 \\
\hline 114710 & 64394 & $\beta \mathrm{Com}$ & 4.23 & 0.57 & 9.2 & G0V & 3.63 & 6030 \\
\hline 116842 & 65477 & $80 \mathrm{UMa}$ & 3.99 & 0.17 & 24.9 & A5V & 0.32 & 8000 \\
\hline 126660 & 70497 & $\theta$ Boo & 4.04 & 0.50 & 14.6 & F7V & 2.95 & 6280 \\
\hline 128167 & 71284 & $\sigma$ Boo & 4.46 & 0.36 & 15.5 & F3Vwvar & 1.70 & 6770 \\
\hline 134083 & 73996 & 45 Boo & 4.93 & 0.43 & 19.7 & F5V & 1.82 & 6500 \\
\hline 139664 & 76829 & g Lup & 4.64 & 0.41 & 17.5 & F5IV-V & 1.12 & 6680 \\
\hline 142373 & 77760 & $\chi$ Her & 4.60 & 0.56 & 15.9 & F9V & 8.51 & 5840 \\
\hline 142860 & 78072 & $\gamma$ Ser & 3.85 & 0.48 & 11.1 & F6V & 3.24 & 6330 \\
\hline 149661 & 81300 & $12 \mathrm{Oph}$ & 5.77 & 0.83 & 9.8 & $\mathrm{~K} 2 \mathrm{~V}$ & 2.09 & 5200 \\
\hline 154088 & 83541 & & 6.59 & 0.81 & 18.1 & K1V & 7.24 & 5000 \\
\hline 156026 & 84478 & & 6.33 & 1.14 & 6.0 & $\mathrm{~K} 5 \mathrm{~V}$ & 0.63 & 4350 \\
\hline 157214 & 84862 & 72 Her & 5.38 & 0.62 & 14.4 & G0V & 7.24 & 5790 \\
\hline 157881 & 85295 & & 7.54 & 1.36 & 7.7 & $\mathrm{~K} 7 \mathrm{~V}$ & 5.25 & 3950 \\
\hline 160691 & 86796 & $\mu$ Ara & 5.12 & 0.69 & 15.3 & G5V & 6.17 & 5750 \\
\hline 161797 & 86974 & $\mu$ Her & 3.42 & 0.75 & 8.4 & G5IV & 4.79 & 5670 \\
\hline
\end{tabular}


Table 2. continued

\begin{tabular}{|c|c|c|c|c|c|c|c|c|}
\hline HD & $\begin{array}{r}\text { HIP } \\
(2)\end{array}$ & $\begin{array}{l}\text { Name } \\
(3)\end{array}$ & $\begin{array}{r}V \\
\text { mag } \\
(4)\end{array}$ & $\begin{array}{r}B-V \\
\text { mag } \\
(5)\end{array}$ & $\begin{array}{r}d \\
\mathrm{pc} \\
(6)\end{array}$ & Spect. & $\begin{array}{r}\text { age } \\
\text { Gyrs } \\
(8)\end{array}$ & $\begin{array}{r}T_{\text {eff }} \\
\mathrm{K} \\
(9)\end{array}$ \\
\hline 166620 & 88972 & & 6.38 & 0.88 & 11.1 & $\mathrm{~K} 2 \mathrm{~V}$ & 7.24 & 4970 \\
\hline 172167 & 91262 & $\alpha$ Lyr & 0.03 & 0.00 & 7.8 & A0Vvar & 0.35 & 9620 \\
\hline 173667 & 92043 & 110 Her & 4.19 & 0.48 & 19.1 & F6V & 2.40 & 6370 \\
\hline 185144 & 96100 & $\sigma$ Dra & 4.67 & 0.79 & 5.8 & KOV & 5.50 & 5330 \\
\hline 185395 & 96441 & $\theta$ Cyg & 4.49 & 0.40 & 18.6 & $\mathrm{~F} 4 \mathrm{~V}$ & 1.29 & 6750 \\
\hline 187642 & 97649 & $\alpha \mathrm{Aql}$ & 0.76 & 0.22 & 5.1 & A7IV-V & 1.23 & 7550 \\
\hline 188512 & 98036 & $\beta \mathrm{Aql}$ & 3.71 & 0.86 & 13.7 & G8IV & 4.27 & 5500 \\
\hline 190248 & 99240 & $\delta \mathrm{Pav}$ & 3.55 & 0.75 & 6.1 & G5IV-Vvar & 5.25 & 5650 \\
\hline 191408 & 99461 & & 5.32 & 0.87 & 6.0 & $\mathrm{~K} 2 \mathrm{~V}$ & 7.24 & 4700 \\
\hline 192310 & 99825 & & 5.73 & 0.88 & 8.8 & $\mathrm{~K} 3 \mathrm{~V}$ & & 5000 \\
\hline 197692 & 102485 & $\psi \mathrm{Cap}$ & 4.13 & 0.43 & 14.7 & F5V & 2.00 & 6540 \\
\hline 198149 & 102422 & $\eta$ Cep & 3.41 & 0.91 & 14.3 & KOIV & 7.94 & 5000 \\
\hline 203280 & 105199 & $\alpha$ Cep & 2.45 & 0.26 & 15.0 & A7IV-V & 0.89 & 7570 \\
\hline 203608 & 105858 & $\gamma \mathrm{Pav}$ & 4.21 & 0.49 & 9.2 & F6V & 10.50 & 6150 \\
\hline 207129 & 107649 & & 5.57 & 0.60 & 15.6 & $\mathrm{G} 2 \mathrm{~V}$ & 6.03 & 5930 \\
\hline 209100 & 108870 & $\zeta$ Ind & 4.69 & 1.06 & 3.6 & K5V & 1.29 & 4600 \\
\hline 215789 & 112623 & $\epsilon$ Gru & 3.49 & 0.08 & 39.8 & $\mathrm{~A} 3 \mathrm{~V}$ & 0.54 & 8420 \\
\hline 216956 & 113368 & $\alpha$ Psa & 1.17 & 0.15 & 7.7 & $\mathrm{~A} 3 \mathrm{~V}$ & 0.22 & 8680 \\
\hline 217014 & 113357 & $51 \mathrm{Peg}$ & 5.45 & 0.67 & 15.4 & G5V & 5.13 & 5810 \\
\hline 219134 & 114622 & & 5.57 & 1.00 & 6.5 & K3Vvar & 12.60 & 4800 \\
\hline 222368 & 116771 & $\iota \mathrm{Psc}$ & 4.13 & 0.51 & 13.8 & F7V & 3.80 & 6190 \\
\hline 222404 & 116727 & $\gamma$ Cep & 3.21 & 1.03 & 13.8 & K1IV & 8.91 & 5000 \\
\hline
\end{tabular}

the responsitivity of the detectors. Similarly, chopping appeared to be an inadequate observing mode at 135 and at $170 \mu \mathrm{m}$ because of confusion with structure in the background from infrared cirrus. We therefore switched to the observing mode PHT22 and made minimaps. Minimaps consumed more observing time and we therefore dropped the observations at 90 and $135 \mu \mathrm{m}$. We tried to reobserve in minimap mode those targets that had already been observed in chopped mode (using extra time allocated when ISO lived longer than expected) but succeeded only partially: several targets had left the observing window. In total we used $65 \mathrm{hrs}$ of observations. In this article we discuss only the stellar flux densities derived from the 60 and $170 \mu \mathrm{m}$ minimaps. Appendix A contains a detailed description of our measurement procedure.

Instrumental problems (mainly detector memory effects) made us postpone the reduction of the chopped measurements until a later date; this applies also to the many (all chopped) measurements at $25 \mu \mathrm{m}$.

We added published (Ábrahám et al. 1998) ISOPHOT measurements of five A-type stars ( $\beta$ UMa, $\gamma \mathrm{UMa}$, $\delta$ UMa, $\epsilon$ UMa and $80 \mathrm{UMa})$. The measurements have been obtained in a different mode from our observations, but we treat all measurements equally. These five stars are all at about 25 pc (Perryman et al. 1997), sufficiently nearby to allow detection of the photospheric flux. These stars are spectroscopic doubles and they do not fulfill all of our selection criteria; below we argue why we included them anyhow. Ábrahám et al. (1998) present ISOPHOT measurements of four more stars, which they assume to be at the same distance because all nine stars are supposed to be members of an equidistant group called the "Ursa Major stream". The Hipparcos measurements
(Perryman et al. 1997), however, show that four of the nine stars are at a distance of $66 \mathrm{pc}$ and thus too far away to be useful for our purposes.

\subsection{Data reduction}

All our data have been reduced using standard calibration tables and the processing steps of OLP6/PIA7. These steps include the instrumental corrections and photometric calibration of the data. At the time when we reduced our data there did not yet exist a standard procedure to extract the flux. We therefore developed and used our own method - see Appendix B.

Later versions of the software which contain upgrades of the photometric calibration do not significantly alter our photometric results and the conclusions of this paper remain unchanged. For each filter the observing mode gave two internal calibration measurements which were closely tuned to the actual sky brightness. This makes the absolute calibration insensitive to instrumental effects as filter-to-filter calibrations and signal non-linearities which were among others the main photometric calibration improvements for the upgrades. In addition, it is standard procedure to ensure that each upgrade does not degrade the photometric calibration of the validated modes of the previous processing version.

\section{Results}

\subsection{Flux densities at $60 \mu \mathrm{m}$}

The flux densities at $60 \mu \mathrm{m}$ are presented in Table 3 . The content of each column is as follows: Col. (1): the HD number; Col. (2): the TDT number as used in the 
Table 3. $60 \mu \mathrm{m}$ data: see text for an explanation of the various columns

\begin{tabular}{|c|c|c|c|c|c|c|c|c|c|}
\hline HD & $\begin{array}{l}\text { ISO_id } \\
(2)\end{array}$ & $\begin{array}{r}F_{\nu} \\
\mathrm{mJy} \\
(3)\end{array}$ & $\begin{array}{r}\sigma_{\nu} \\
\mathrm{mJy} \\
(4)\end{array}$ & $\begin{array}{r}F_{\nu}^{\text {pred }} \\
\text { mJy } \\
(5)\end{array}$ & $\begin{array}{r}F_{\nu}^{\operatorname{exc}} \\
\mathrm{mJy} \\
(6)\end{array}$ & $\begin{array}{r}F_{\nu}^{\operatorname{exc}} / \sigma_{\nu} \\
(7) \\
\end{array}$ & $\begin{array}{r}F_{\nu}^{\text {disk }} \\
\text { mJy } \\
(8) \\
\end{array}$ & $\begin{array}{r}\log \tau_{60}^{\text {disk }} \\
(9) \\
(9)\end{array}$ & $\begin{array}{l}\text { Reference } \\
\text { (10) }\end{array}$ \\
\hline 693 & 74900501 & $\overline{33}$ & 34 & 43 & -10 & $\overline{<1}$ & & $<-4.6$ & ISO minimap \\
\hline 1581 & 69700102 & 94 & 19 & 94 & 0 & $<1$ & & $<-5.0$ & ISO minimap \\
\hline 2151 & & 351 & 100 & 376 & -25 & $<1$ & & & IFSC \\
\hline 4628 & 61901104 & 41 & 24 & 44 & -4 & $<1$ & & $<-4.1$ & ISO minimap \\
\hline 4813 & 61901705 & 55 & 37 & 34 & 20 & $<1$ & & $<-4.3$ & ISO minimap \\
\hline 7570 & 72002506 & 85 & 25 & 47 & 38 & 1.6 & & $<-4.4$ & ISO minimap \\
\hline 9826 & 61503786 & 100 & 22 & 98 & 2 & $<1$ & & $<-5.0$ & ISO minimap \\
\hline 10700 & 75701121 & 433 & 37 & 253 & 180 & 4.9 & 190 & -4.6 & ISO minimap \\
\hline 10780 & 61503507 & 43 & 30 & 41 & 3 & $<1$ & & $<-4.1$ & ISO minimap \\
\hline 12311 & 69100108 & 189 & 18 & 178 & 10 & $<1$ & & $<-5.6$ & ISO minimap \\
\hline 13445 & 73100771 & 25 & 24 & 26 & -2 & $<1$ & & $<-4.1$ & ISO minimap \\
\hline 14412 & 76301673 & -12 & 14 & 18 & -30 & $<1$ & & $<-4.4$ & ISO minimap \\
\hline 14802 & 80201174 & 56 & 19 & 41 & 14 & $<1$ & & $<-4.5$ & ISO minimap \\
\hline 15008 & 71801511 & 41 & 9 & 30 & 10 & 1.1 & & $<-5.4$ & ISO minimap \\
\hline 17051 & 76500413 & 45 & 15 & 31 & 14 & $<1$ & & $<-4.5$ & ISO minimap \\
\hline 17925 & 78100314 & 104 & 24 & 31 & 73 & 3.1 & 80 & -3.9 & ISO minimap \\
\hline 19373 & 81001847 & 122 & 13 & 116 & 6 & $<1$ & & $<-5.2$ & ISO minimap \\
\hline 20630 & 79201553 & 9 & 33 & 66 & -57 & $<1$ & & $<-4.9$ & ISO minimap \\
\hline 20766 & 69100715 & 34 & 24 & 32 & 2 & $<1$ & & $<-4.4$ & ISO minimap \\
\hline 20807 & 57801756 & 30 & 13 & 39 & -9 & $<1$ & & $<-4.9$ & ISO minimap \\
\hline 22001 & 69100659 & 52 & 9 & 42 & 9 & 1.0 & & $<-5.1$ & ISO minimap \\
\hline 22049 & & 1250 & 100 & 278 & 967 & 9.7 & 1260 & & IFSC \\
\hline 22484 & 79501562 & 141 & 27 & 89 & 51 & 1.9 & & $<-4.6$ & ISO minimap \\
\hline 23249 & & 270 & 100 & 363 & -92 & $<1$ & & & IFSC \\
\hline 26965 & 84801865 & 121 & 21 & 128 & -7 & $<1$ & & $<-4.7$ & ISO minimap \\
\hline 30495 & 83901668 & 174 & 31 & 33 & 141 & 4.5 & 150 & -4.1 & ISO minimap \\
\hline 33262 & 58900871 & 81 & 21 & 55 & 26 & 1.3 & & $<-4.7$ & ISO minimap \\
\hline 34411 & 83801474 & 63 & 13 & 69 & -5 & $<1$ & & $<-5.0$ & ISO minimap \\
\hline 37394 & 83801977 & 40 & 13 & 26 & 14 & 1.0 & & $<-4.0$ & ISO minimap \\
\hline 38392 & 70201402 & 31 & 24 & 34 & -2 & $<1$ & & $<-3.9$ & ISO minimap \\
\hline 38393 & 70201305 & 160 & 25 & 138 & 22 & $<1$ & & $<-5.1$ & ISO minimap \\
\hline 38678 & 69202308 & 349 & 22 & 60 & 289 & 13.3 & 310 & -4.7 & ISO minimap \\
\hline 39060 & 70201080 & 14700 & 346 & 54 & 14650 & 42.4 & 15500 & -2.8 & ISO minimap \\
\hline 43834 & 62003217 & 48 & 16 & 56 & -8 & $<1$ & & $<-4.8$ & ISO minimap \\
\hline 48915 & 72301711 & 4230 & 155 & 4650 & -420 & $<1$ & & $<-7.5$ & ISO minimap \\
\hline 50281 & 71802114 & -4 & 16 & 30 & -33 & $<1$ & & $<-4.3$ & ISO minimap \\
\hline 61421 & & 2290 & 100 & 2350 & -59 & $<1$ & & $<-6.0$ & IFSC \\
\hline 74956 & & 399 & 100 & 226 & 173 & 1.7 & & & IPSC \\
\hline 75732 & 17800102 & 160 & 28 & 35 & 126 & 4.4 & 130 & -3.8 & Dominik et al. 1998 \\
\hline 80007 & & 284 & 100 & 311 & -27 & $<1$ & & & IFSC \\
\hline 95418 & 19700563 & 539 & 135 & 152 & 387 & 2.9 & 410 & -5.0 & Ábrahám et al. 1998 \\
\hline 102647 & & 784 & 100 & 213 & 571 & 5.7 & 750 & -4.8 & IFSC \\
\hline 102870 & & 137 & 100 & 150 & -14 & $<1$ & & $<-4.6$ & IFSC \\
\hline 103287 & 19500468 & 164 & 41 & 147 & 17 & $<1$ & & $<-5.5$ & Ábrahám et al. 1998 \\
\hline 106591 & 19700973 & 94 & 59 & 69 & 25 & $<1$ & & $<-4.9$ & Ábrahám et al. 1998 \\
\hline 110833 & 60000526 & -7 & 14 & 15 & -22 & $<1$ & & & ISO minimap \\
\hline 112185 & 34600578 & 322 & 81 & 223 & 99 & 1.2 & & $<-5.4$ & Ábrahám et al. 1998 \\
\hline 114710 & 61000119 & 106 & 34 & 93 & 13 & $<1$ & & $<-4.7$ & ISO minimap \\
\hline 116842 & 19500983 & 40 & 34 & 47 & -7 & $<1$ & & & Ábrahám et al. 1998 \\
\hline 126660 & 61000834 & 93 & 21 & 95 & -2 & $<1$ & & $<-5.1$ & ISO minimap \\
\hline 128167 & 61001236 & 100 & 19 & 48 & 52 & 2.8 & 55 & -5.0 & ISO minimap \\
\hline 134083 & 61001337 & 70 & 26 & 36 & 34 & 1.3 & & $<-4.5$ & ISO minimap \\
\hline 139664 & 64700880 & 488 & 48 & 45 & 442 & 9.2 & 470 & -4.0 & ISO minimap \\
\hline 142373 & 61001139 & 71 & 21 & 64 & 6 & $<1$ & & $<-4.8$ & ISO minimap \\
\hline 142860 & 63102981 & 113 & 24 & 109 & 4 & $<1$ & & $<-5.1$ & ISO minimap \\
\hline 149661 & 80700365 & 56 & 22 & 38 & 18 & $<1$ & & $<-4.1$ & ISO minimap \\
\hline 154088 & 64702041 & 40 & 55 & 17 & 22 & $<1$ & & $<-3.3$ & ISO minimap \\
\hline 156026 & 64702142 & 30 & 34 & 43 & -13 & $<1$ & & $<-3.4$ & ISO minimap \\
\hline 157214 & 71000144 & 27 & 26 & 36 & -8 & $<1$ & & $<-4.4$ & ISO minimap \\
\hline 157881 & 65000845 & 41 & 22 & 24 & 17 & $<1$ & & $<-2.6$ & ISO minimap \\
\hline 160691 & 64402347 & 73 & 18 & 52 & 21 & 1.2 & & $<-4.5$ & ISO minimap \\
\hline 161797 & & 222 & 100 & 281 & -59 & $<1$ & & $<-4.6$ & IFSC \\
\hline
\end{tabular}


Table 3. continued

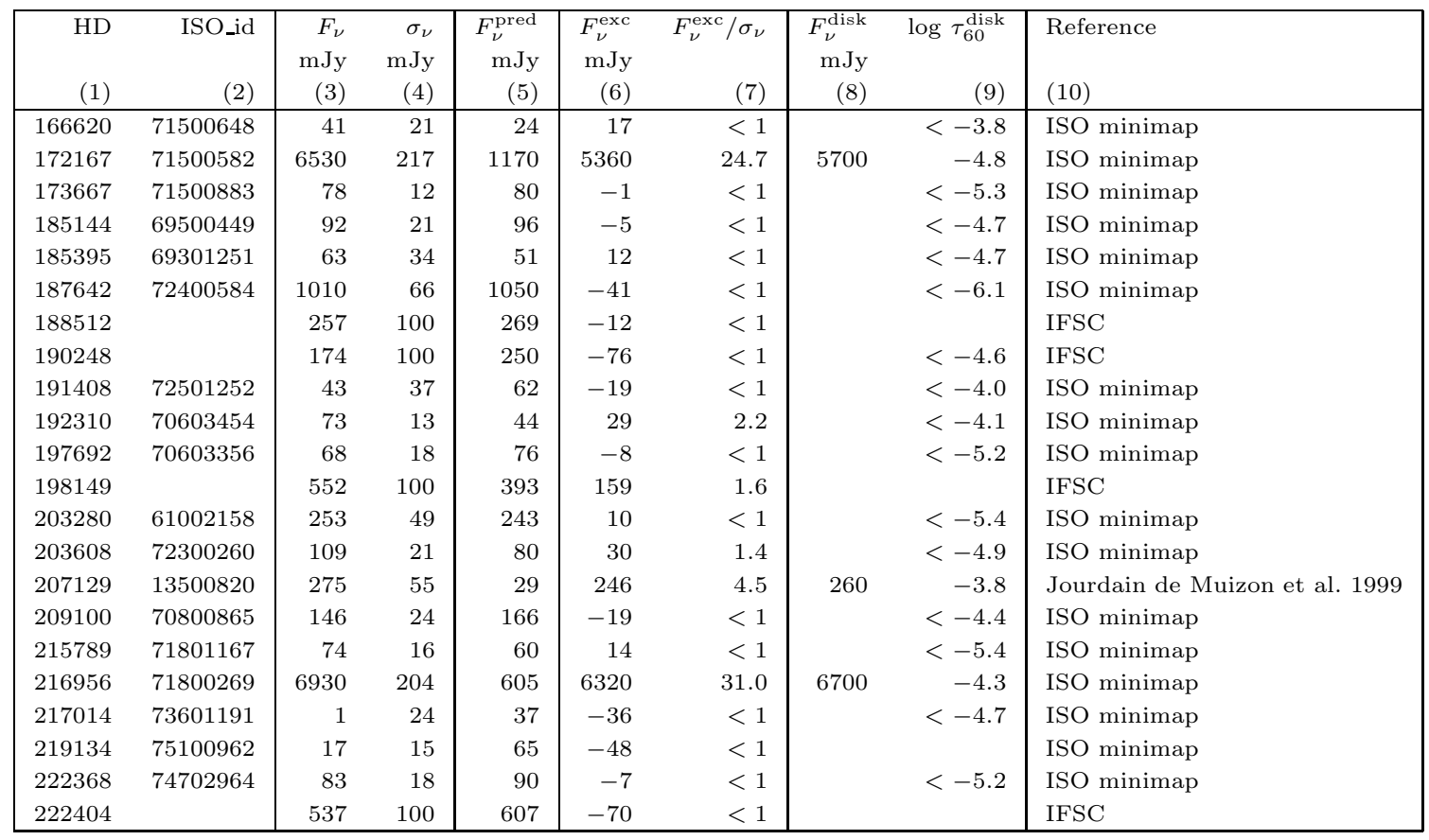

ISO archive; Col. (3) the flux density corrected for bandwidth effects (assuming that the spectrum is characterized by the Rayleigh-Jeans equation) and for the fact that the stellar flux extended over more than 1 pixel; Col. (4) the error estimate assigned by the ISOPHOT software to the flux measurement in Col. (3); for the IRAS measurements the error has been put at $100 \mathrm{mJy}$; Col. (5) the flux expected from the stellar photosphere, $F_{\nu}^{\text {pred }}$ as derived from Eq. (1) using the $V$ and $(B-V)$ values in Table 2; Col. (6): the difference between Cols. (3) and (5); we call it the "excess flux", $F_{\nu}^{\text {exc }} ;(7)$ : the ratio of the excess flux compared to the measurement error given in Col. (4); when we concluded that the excess is real and not a measurement error we recalculated the monochromatic flux density by assuming a flat spectrum within the ISOPHOT $60 \mu \mathrm{m}$ bandwidth; the result is in Col. (8) and is called $F_{\nu}^{\text {disk }}$. Column $(9)$ shows an estimate of $\tau_{60}^{\text {disk }}$, the optical depth of the disk at visual wavelengths, but estimated from the flux density at $60 \mu \mathrm{m}$; see below for a definition and see Appendix D for more details. Flux densities in Cols. (3), (4), (6) and (7) have been corrected for the point spread function being larger than the pixel size of the detector and for Rayleigh-Jeans colour-correction (cc); for ISO fluxes, the inband flux has been divided by 0.69 (the correction for the point spread function (= psf), see Appendix B) and by 1.06 (cc) and for IRAS fluxes, the IFSC or IPSC flux have been divided by 1.31 (cc). The "disk emission" in Col. (8) is "de-colour-corrected" from Col. (6).

We have checked the quality of our results at $60 \mu \mathrm{m}$ in two ways: (i) by comparing ISO with IRAS flux densities; (ii) by comparing fluxes measured by ISO with predictions

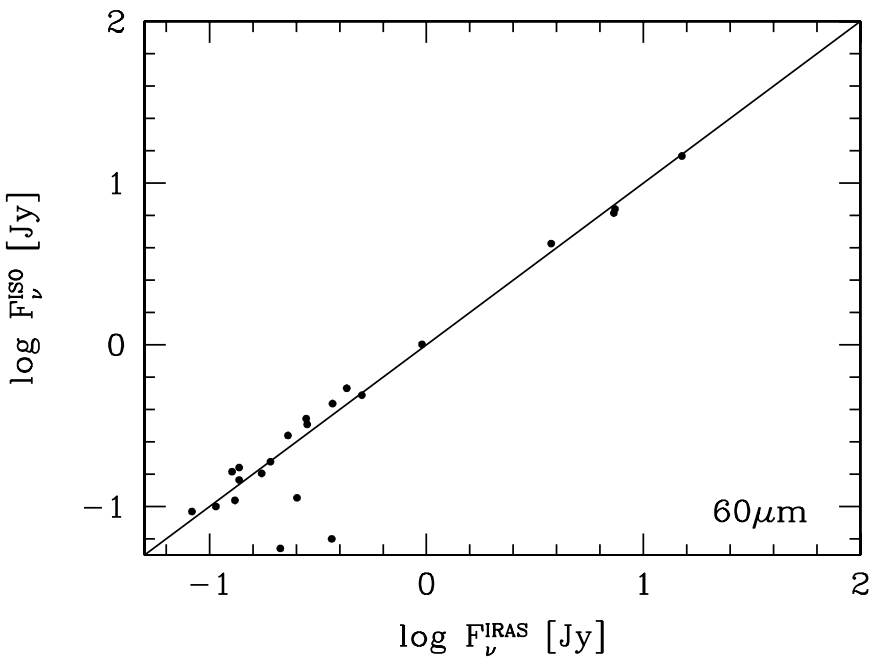

Fig. 1. Correlation of fluxes measured by IRAS and by ISO, respectively. The line marks the relation $F_{\nu}^{\mathrm{IRAS}}=F_{\nu}^{\mathrm{ISO}}$

based on the $(B-V)$ photometric index. The second approach allows us to assess the quality of ISO flux densities below the IRAS sensitivity limit.

\subsection{The correlation between IRAS and ISO measurements at $60 \mu \mathrm{m}$}

Figure 1 shows the strong correlation between IRAS and ISO $60 \mu \mathrm{m}$ flux densities down to about the $60 \mathrm{mJy}$ level of ISO. For three of the fainter stars the IRAS fluxes are considerably higher than those of ISO. For one of these three, HD 142860, the ISO measurements show the presence of two nearby $60 \mu \mathrm{m}$ sources; the larger IRAS beam 
Table 4. Flux densities measured at $170 \mu \mathrm{m}$. The various columns have the same meaning as in the previous table

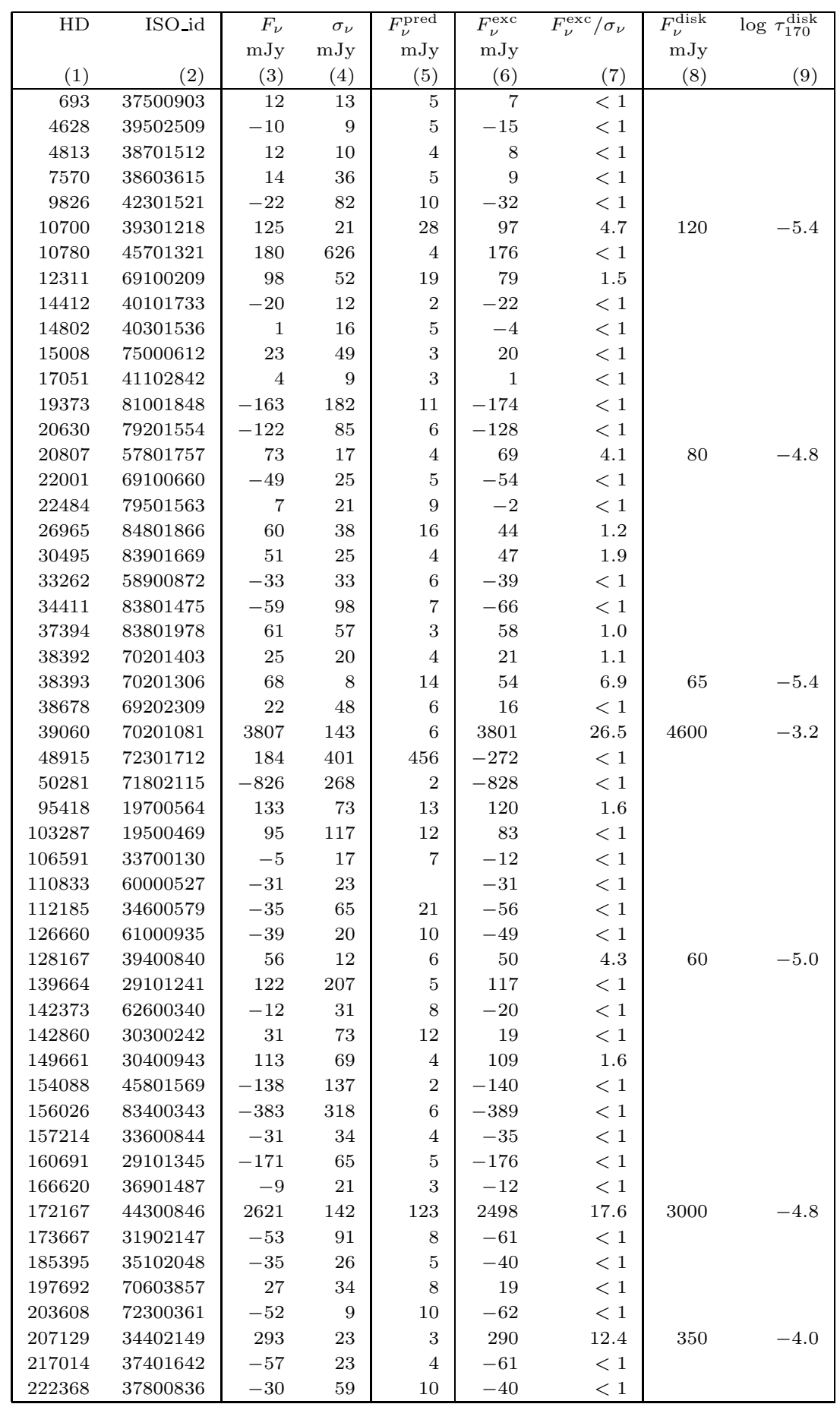

Note: Fluxes in Cols. 3, 4, 6, 7 are corrected for point spread function and Rayleigh-Jeans colour-correction, i.e. the inband flux has been divided by 0.64 (psf) and by 1.2 (cc). The "Excess" in Col. 8 is "de-colour-corrected" from Col. 6.

has merged the three sources; see Fig. 4. For the two remaining sources in Fig. 1 with different IRAS and ISO flux densities we assume that the IRAS measurement is too high because noise lifted the measured flux density above the detection limit, a well-known effect for measurements close to the sensitivity limit of a telescope.

\subsection{The correlation between predicted and measured flux densities at $60 \mu \mathrm{m}$}

A strong correlation exists between predicted and measured flux density, Cols. (8) and (3) in Table 3, as seen in Fig. 2. 


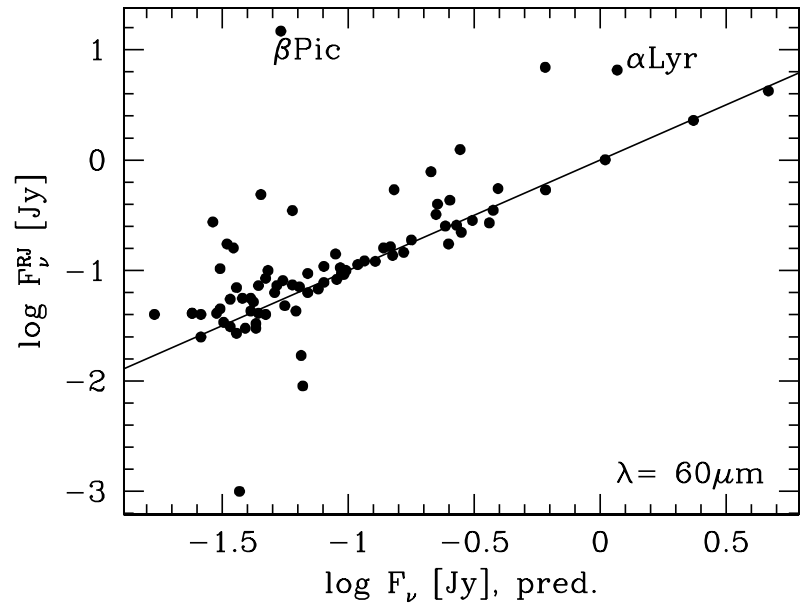

Fig. 2. Diagram of predicted and measured fluxes at $60 \mu \mathrm{m}$. The predicted fluxes were derived mainly from Eq. (1) except in a few cases where Kurucz model atmospherese were fitted to photometric points at optical wavelengths. See text. The line marks the relation $F_{\nu}=F_{\nu}^{\text {pred }}$

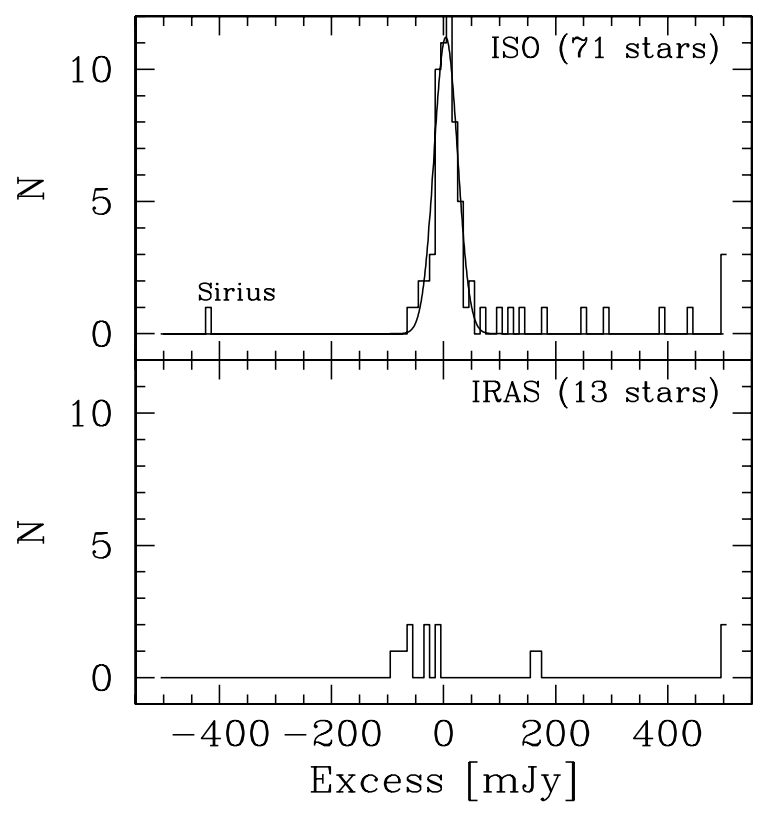

Fig. 3. Histogram of the differences between the measured flux density and the one predicted at $60 \mu \mathrm{m}$. Top: distribution of the flux densities measured by ISO; there are three stars with an excess higher than $500 \mathrm{mJy}$; the drawn curve is a Gauss curve with average $\mu=4 \mathrm{mJy}$ and dispersion $\sigma=21 \mathrm{mJy}$. Bottom: the same for stars where only IRAS data are available; two stars have an excess higher than $500 \mathrm{mJy}$

Figure 3 shows that the distribution of the excess fluxes can be split into two components: a very narrow distribution around zero plus a strong wing of positive excesses, i.e. cases where we measure more flux than is produced by the stellar photosphere. In these cases a disk is very probably present. A Gauss curve has been drawn in the figure with parameters $\mu=4 \mathrm{mJy}$ and $\sigma=$ $21 \mathrm{mJy}$, where $\mu$ is the average and $\sigma$ the dispersion. The value of $\sigma$ agrees with the magnitude of individual error

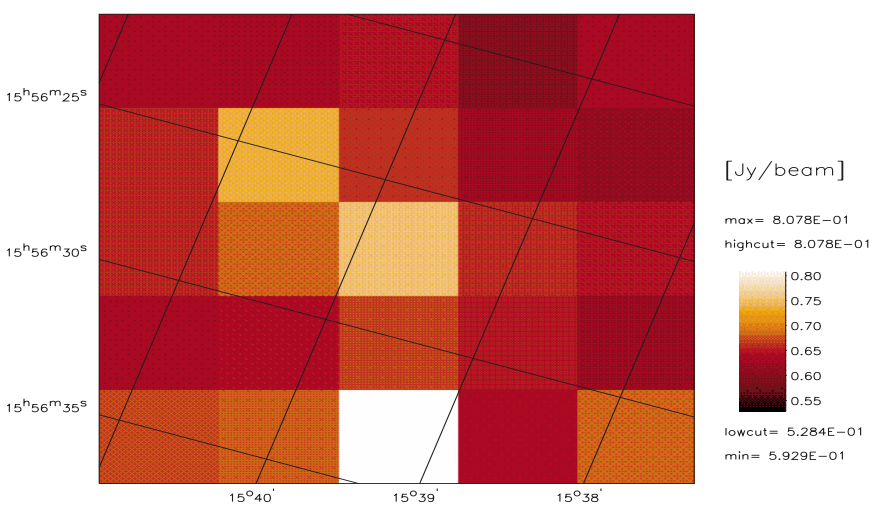

Fig. 4. The $60 \mu \mathrm{m}$ image in spacecraft orientation of the region around HD 142860 as obtained from the ISOPHOT minimap. There are three point sources in the field, the position of the source in the centre corresponds to the position of HD 142860. The upper source has $F_{\nu}=140 \pm 40 \mathrm{mJy}$ and coordinates (J2000) RA $15^{\mathrm{h}} 56^{\mathrm{m}} 25^{\mathrm{s}}$, Dec $15^{\circ} 40^{\prime} 43^{\prime \prime}$; the lower source has $F_{\nu}=250 \pm 40 \mathrm{mJy}$ and coordinates $(\mathrm{J} 2000) \mathrm{RA} 15^{\mathrm{h}} 56^{\mathrm{m}} 33^{\mathrm{s}}$, Dec. $15^{\circ} 39^{\prime} 15^{\prime \prime}$

measurements as given in Col. 8 in Table 3. The one case in Fig. 3 with a strong negative excess, i.e. where we measured less than is predicted, concerns Sirius, $\alpha$ CMa; we interpret this negative excess as a consequence of the poor correction for transient effects of the detector for this strong infrared source.

We have concluded that a disk is present when $F_{\nu}^{\text {exc }}>$ $\mu+3 \sigma=65$ mJy. A summary of data on all stars with disks is in Table 6. HD 128167 has also been labelled as a "detection" although $F_{\nu}^{\text {exc }}$ is only $2.8 \sigma_{\nu}$. The star is one of the few with a detection at $170 \mu \mathrm{m}$ and this removed our doubts about the detection at $60 \mu \mathrm{m}$.

\subsection{Results at $170 \mu \mathrm{m}$}

The results are shown in Table 4 which has the same structure as Table 3. IRAS did not measure beyond $100 \mu \mathrm{m}$ and we have no existing data to compare with our ISO data. Neither can we make a useful comparison between measured and predicted flux densities because the photospheric flux is expected to be roughly $1 / 8$ of the $60 \mu \mathrm{m}$ flux density and for most stars this is below the sensitivity limit of ISO.

We accepted fluxes as real when $F_{\nu}^{\text {exc }}>3 \sigma_{\nu}$ and the minimap showed flux only in the pixel illuminated by the star. This leads to seven detections at $170 \mu \mathrm{m}$ in Table 4 . All seven stars have excess emission also at $60 \mu \mathrm{m}$ except HD 20807 and HD 38393. Very probably these last two stars have accidentally been misidentified with unrelated background sources, as we will show now.

\subsection{Should some detections be identified with unrelated field sources?}

We need to consider the possible influence of the fieldsource population upon our results. 
Table 5. Probability of spurious detections

\begin{tabular}{|rrrrrrr|}
\hline$\lambda$ & $\begin{array}{c}F_{\nu}^{\lim } \\
\mu \mathrm{m}\end{array}$ & $p$ & $q$ & $P(q, 1)$ & $P(q, 2)$ & $P(q, 3)$ \\
\hline 60 & 100 & 0.006 & 84 & 0.397 & 0.091 & \\
60 & 150 & 0.0015 & 84 & 0.117 & 0.007 & \\
60 & 200 & 0.001 & 84 & 0.079 & 0.003 & \\
170 & 50 & 0.15 & 52 & 1.000 & 0.997 & 0.988 \\
170 & 100 & 0.07 & 52 & 0.975 & 0.881 & 0.702 \\
170 & 200 & 0.04 & 52 & 0.875 & 0.61 & 0.334 \\
170 & 300 & 0.005 & 52 & 0.226 & 0.027 & \\
170 & 1000 & 0.00025 & 52 & 0.013 & & \\
\hline
\end{tabular}

Dole et al. (2000), Matsuhara et al. (2000), Oliver et al. (2000) and Elbaz et al. (2000) quote source counts at respectively 170,90 and $15 \mu \mathrm{m}$ from which the surface density of sources on the sky can be read down to the sensitivity limits of our measurements. In the 170 and $90 \mu \mathrm{m}$ cases this involves the authors' extrapolations, via models, of their source counts from the roughly 100-200 mJy flux limits of their respective datasets. At $60 \mu \mathrm{m}$ source counts can be approximated, with sufficient accuracy for present purposes, by interpolation from the other wavelengths. Using these source densities we now estimate the probability that our samples of detections contain one or two field sources unrelated to the star in question.

Since we know, for all of our targets, into exactly which pixel of the PHT map they should fall, we need to consider the probability that a field source with flux down to our sensitivity limit falls into the relevant PHT pixel. This effective "beam" area is $45^{\prime \prime} \times 45^{\prime \prime}$ and $100^{\prime \prime} \times 100^{\prime \prime}$, at 60 and $170 \mu \mathrm{m}$ respectively.

At $60 \mu \mathrm{m}$ we have explored 84 beams (targets) and at $170 \mu \mathrm{m} 52$ beams (targets). We apply the binomial distribution to determine the probability $P(q, r)$ that at least $r$ spurious detections occur in $q$ trials when the probability per observation equals $p$.

Table 5 lists the following parameters: Col. (1) shows the wavelength that we consider; Col. (3) contains the probability $p$ to find a source in any randomly chosen pixel with a flux density above the limit $F_{\nu}^{\lim }$ given in Col. (2). Column (4) lists the number $q$ of targets (i.e. trials) in the 60 and $170 \mu \mathrm{m}$ samples. Columns (5)-(7) give the probability $P(q, r)$ of finding at least 1,2 and 3 spurious detections with $F_{\nu}>F_{\nu}^{\lim }$ within a sample of size $q$.

\subsubsection{Probability of spurious detections at $60 \mu \mathrm{m}$}

It follows from Table 5 that there is a $40 \%$ chance that at least one of the two detections at $60 \mu \mathrm{m}$ below $100 \mathrm{mJy}$ is due to a field source, and there is a $9 \%$ chance that both are.

If we ignore field sources and consider the likelihood of spurious excesses occurring above a $3 \sigma$ detection-limit due purely to statistical fluctuations in the measurements, we find that random noise contributes (coincidentally) a further 0.006 spurious detections per beam, on average, for the faintest detections (near $3 \sigma$ ).

The cumulative probability, therefore, is 0.64 that at least 1 of the two detections at $60 \mu \mathrm{m}$ below $100 \mathrm{mJy}$ is not related to a disk; the probability is 0.17 that they are both spurious.

\subsubsection{Probability of spurious detections at $170 \mu \mathrm{m}$}

Table 4 lists 3 detections below 100 mJy. Two (HD 20630 and 38393) have not been detected at $60 \mu \mathrm{m}$. Table 5 shows that the probability is high that both are background sources unrelated to the two stars in question. In the further discussion these two stars have been considered to be without a disk. The third source with a $170 \mu \mathrm{m}$ flux density below $100 \mathrm{mJy}$, HD 128167, has been detected also at $60 \mu \mathrm{m}$. We assume that this detection is genuine and that the source coincides with the star. The remaining four detections at $170 \mu \mathrm{m}$ with $F_{\nu}>100 \mathrm{mJy}$ are also correctly identified with the appropriate star.

\section{Discussion}

Data for the stars with a disk are summarized in Table 6 . Each column contains quantities defined and used in earlier tables with the exception of the last column that contains an estimate of the mass of the disk, $M_{\mathrm{d}}$.

\subsection{Comparison with the IRAS heritage}

The overall good agreement between the IRAS and ISO measurements has already been discussed. The IRAS data base has been explored by several different groups in search of more Vega-like stars. Backman \& Paresce (1993) have reviewed most of these searches. Their Table X contains all stars with disks. For nine of those in Table $\mathrm{X}$ we have ISO measurements, and in eight cases these show the presence of a disk. The one exception is $\delta$ Vel, HD 74956 . We find excess emission but the excess is insignificant (only $1.7 \sigma$ ) and we did not include the star in Table 6 . Thus our data agree well with those discussed by Backman \& Paresce (1993). Although ISO was more sensitive than IRAS at $60 \mu \mathrm{m}$ by about a factor of 5 we have only one new detection of a disk: HD 17925, with an excess of $82 \mathrm{mJy}$ at $60 \mu \mathrm{m}$.

Plets \& Vynckier (1999) have analysed IRAS data in search of "Vega-like" stars and paid special attention to our list of candidate stars. In general there is good agreement between their conclusions and ours. Nine of the disk stars they find in IRAS we confirm with our ISO data. Four of the stars for which they find evidence of a disk at $60 \mu \mathrm{m}$ are without excess emission in our sample. In two cases (HD 142860 and HD 215789) the difference IRAS/ISO is large: IRAS: 413 and $220 \mathrm{mJy}$; ISO: 113 and $78 \mathrm{mJy}$ ). In both cases we are of the opinion that at such low flux levels the ISO data are to be preferred over the IRAS data. 
Table 6. The $60 \mu \mathrm{m}$ excess stars

\begin{tabular}{|c|c|c|c|c|c|c|c|c|c|c|c|}
\hline $\mathrm{HD}$ & $\begin{array}{r}\text { Name } \\
(2)\end{array}$ & $\begin{array}{r}\text { Spect. } \\
\text { (3) }\end{array}$ & $\begin{array}{r}\text { age } \\
\text { Gyrs } \\
(4)\end{array}$ & $\begin{array}{r}F_{\nu} \\
\mathrm{mJy} \\
(5)\end{array}$ & $\begin{array}{r}\sigma_{\nu} \\
\mathrm{mJy} \\
(6)\end{array}$ & $\begin{array}{r}0 \mu \mathrm{m} \\
F_{\nu}^{\text {disk }} \\
\text { mJy } \\
(7)\end{array}$ & $\begin{array}{r}{ }^{10} \log \tau_{60}^{\text {disk }} \\
\text { (8) }\end{array}$ & $\begin{array}{r}F_{\nu} \\
\mathrm{mJy} \\
(9)\end{array}$ & $\begin{array}{r}170 \mu \mathrm{1} \\
\sigma_{\nu} \\
\mathrm{mJy} \\
(10)\end{array}$ & $\begin{array}{c}F_{\nu}^{\text {disk }} \\
\text { mJy } \\
(11)\end{array}$ & $\begin{array}{r}M_{\mathrm{d}} \\
10^{-5} M_{\oplus} \\
(12)\end{array}$ \\
\hline$\frac{1}{10700}$ & $\tau$ Cet & G8V & 7.24 & 433 & 37 & 190 & -4.6 & 125 & 21 & 120 & 20 \\
\hline 17925 & & $\mathrm{~K} 1 \mathrm{~V}$ & 0.08 & 104 & 24 & 80 & -3.9 & & & & 108 \\
\hline 22049 & $\epsilon$ Eri & $\mathrm{K} 2 \mathrm{~V}$ & 0.33 & 1250 & 100 & 1260 & & & & & \\
\hline 30495 & 58 Eri & G3V & 0.21 & 174 & 31 & 150 & -4.1 & 51 & 25 & & 73 \\
\hline 38678 & $\zeta$ Lep & A 2 Vann & 0.37 & 349 & 22 & 310 & -4.7 & 22 & 48 & & 18 \\
\hline 39060 & $\beta \mathrm{Pic}$ & $\mathrm{A} 3 \mathrm{~V}$ & 0.28 & 14700 & 346 & 15500 & -2.8 & 3810 & 143 & 4600 & 1200 \\
\hline 75732 & $\rho^{1} \mathrm{Cnc}$ & G8V & 5.01 & 160 & 28 & 130 & -3.8 & & & & 130 \\
\hline 95418 & $\beta \mathrm{UMa}$ & $\mathrm{A} 1 \mathrm{~V}$ & 0.36 & 539 & 135 & 410 & -5.0 & 133 & 290 & & 8 \\
\hline 102647 & $\beta$ Leo & A3V & 0.24 & 784 & 100 & 750 & -4.8 & & & & 13 \\
\hline 128167 & $\sigma \mathrm{Boo}$ & F3Vwvar & 1.70 & 100 & 19 & 55 & -5.0 & 56 & 12 & 60 & 8 \\
\hline 139664 & g Lup & F5IV-V & 1.12 & 488 & 48 & 470 & -4.0 & 122 & 830 & & 84 \\
\hline 172167 & $\alpha \mathrm{Lyr}$ & A0Vvar & 0.35 & 6530 & 217 & 5700 & -4.8 & 2620 & 142 & 3000 & 13 \\
\hline 207129 & & G2V & 6.03 & 275 & 55 & 260 & -3.8 & 293 & 23 & 350 & 132 \\
\hline 216956 & $\alpha \mathrm{PsA}$ & A3V & 0.22 & 6930 & 204 & 6700 & -4.3 & & & & 44 \\
\hline
\end{tabular}

Note: Fluxes in Cols. 5, 6, 9, 10 are corrected for point spread function and Rayleigh-Jeans colour-correction. Excesses in Col. 7 and 11 are "de-colour-corrected" from Cols. 5 and 9 respectively (see text).

\subsection{Differences with disks around pre-main-sequence stars}

Disks have been found around many pre-main-sequence (= PMS $)$ stars; here we discuss disks around mainsequence $(=$ MS) stars. Disks around PMS stars are always detected by their molecular line emission; they contain dust and gas. The search for molecular emission lines in MS disks, however, has been fruitless so far (Liseau 1999). This is in line with model calculations by Kamp \& Bertoldi (2000) who show that CO in disks around MSstars will be dissociated by the interstellar radiation field. Recent observations with ISO indicate the presence of $\mathrm{H}_{2}$ in the disk around $\beta$ Pic and HST spectra show the presence of CO absorption lines (van Dishoeck, private communication), but the disk around $\beta$ Pic is probably much "fatter" than those around our MS-stars; it is not even certain that $\beta$ Pic is a PMS or MS-star. We will henceforth assume that disks detected around MS-stars contain only dust and no gas.

\subsection{A simple quantitative model}

We have very little information on the disks: in most cases only the photometric flux at $60 \mu \mathrm{m}$. For the quantitative discussion of our measurements we will therefore use a very simple model. We assume a main-sequence star with an effective temperature $T_{\text {eff }}$ and a luminosity $L_{\star}$. The star is surrounded by a disk of $N$ dust particles. For simplicity, and to allow an easy comparison between different stars, we use a unique distance of the circumstellar dust of $r=50 \mathrm{AU}$. This value is consistent with the measurements of spatially resolved disks like Vega and $\epsilon$ Eri and also with the size of the Kuiper Belt in our own solar system. The particles are spherical, have all the same diameter and are made of the same material. The important parameter of the disk that varies from star to star is $N$. The temperature, $T_{\mathrm{d}}$, of each dust particle is determined by the equilibrium between absorption of stellar photons and by emission of infrared photons; thus $T_{\mathrm{d}}$ depends on $T_{\text {eff }}$.

Each dust particle absorbs photons with an effective cross section equal to $Q_{\nu} \pi a^{2} ; Q_{\nu}$ is the absorption efficiency of the dust and $a$ the radius of a dust particle. The average of $Q_{\nu}$ over the Planck function will be written as $Q_{\text {ave }}$. The dust particles absorb a fraction $\tau \equiv N Q_{\text {ave }}\left(T_{\text {eff }}\right) a^{2} /\left(4 r^{2}\right)$ of the stellar energy and reemit this amount of energy in the infrared; in all cases the value of $\tau$ is very small. We will call $\tau$ the "optical depth of the disk"; it represents the extinction by the disk at visual wavelengths:

$\tau=\frac{L_{\mathrm{d}}}{L_{*}}=\frac{F_{\mathrm{bol}}^{\mathrm{d}}}{F_{\mathrm{bol}}^{\text {pred }}}$.

The mass of the disk, $M_{\mathrm{d}}$, is proportional to $\tau$ :

$M_{\mathrm{d}}=\frac{16 \pi}{3} \frac{\rho a r^{2}}{Q_{\text {ave }}} \tau$.

We will use spheres with a radius $a$ of $1 \mu \mathrm{m}$ and with material density $\rho$ and optical constants of interstellar silicate (Draine \& Lee 1984); we use $Q_{\text {ave }}=0.8$ and derive $M_{\mathrm{d}}=0.5 \tau \mathrm{M}_{\oplus}$. It is known that the grains in Vega-like systems are much larger than interstellar grains. For A stars, the emission is probably dominated by grains larger than $10 \mu \mathrm{m}$ (Aumann et al. 1984; Zuckerman \& Becklin 1993; Chini et al. 1991), because smaller grains are blown out by radiation pressure. However, for F, G, and K stars, the blowout sizes are $1 \mu \mathrm{m}$ or smaller and it must be assumed that the emission from these stars is dominated by smaller grains. We calculate mass estimates using the grain size of $1 \mu \mathrm{m}$. Since the mass estimates depend linearly upon the grain size, the true masses of systems with bigger grains can easily be calculated by scaling the value. The mean absorption efficiency factor $Q_{\text {ave }}$ is only weakly dependent upon the grain size for sizes between 1 and $100 \mu \mathrm{m}$.

Numerical simulations made us discover a simple property of this model that is significant because it makes the 


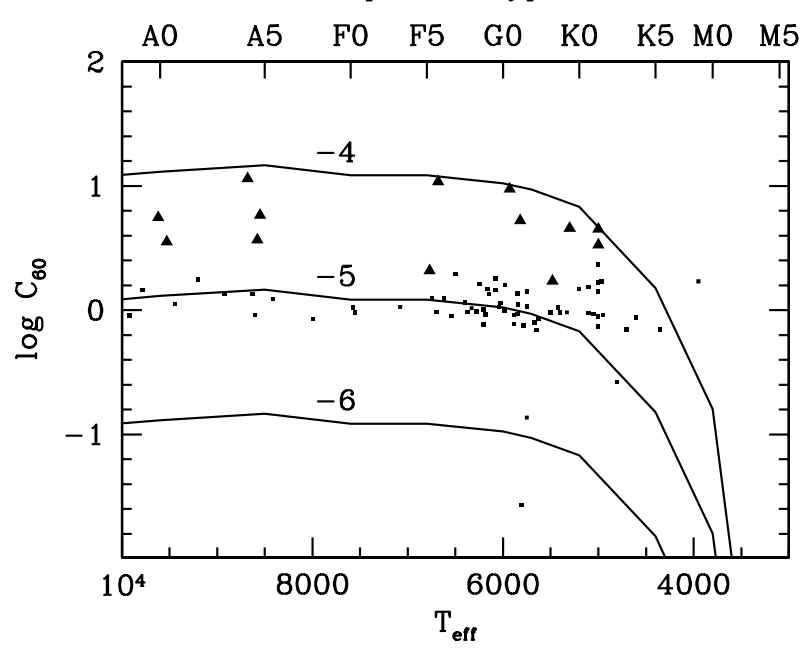

Fig. 5. The ${ }^{10}$ logarithm of the fraction of stellar energy emitted by the disk is shown as a function of $T_{\text {eff }}$ and $C_{60}$. The labels to the curves indicate the ${ }^{10} \operatorname{logarithm}$ of $\tau$. The triangles represent stars with a disk, and the small squares indicate the upper limit of non-detections. Using our standard dust particle model the mass of each disk is given by $M_{\mathrm{d}}=0.5 \tau M_{\oplus}$ (see text)

detection probability constant for disks of stars of different spectral type . Define a variable called "contrast": $C_{60} \equiv\left(L_{\nu, \mathrm{d}} / L_{\nu, *}\right)_{60 \mu \mathrm{m}}$, and assume black body radiation by the star and by the dust particles, then $C_{60}$ is constant for $T_{\text {eff }}$ in the range of $\mathrm{A}, \mathrm{F}$, and G-stars. The reason for this constancy is that when $T_{\text {eff }}$ drops the grains get colder and emit less in total, but because $60 \mu \mathrm{m}$ is at the Wien side of the Planck curve, their emission rate at $60 \mu \mathrm{m}$ goes up. For a more elaborate discussion see Appendix C. Let us then make a two-dimensional diagram of the values of $\tau$ (or of $M_{\mathrm{d}}$ ) as a function of $T_{\text {eff }}$ and $C_{60}$ : see Fig. 5. Constant values of $\tau$ appear as horizontal contours for $T_{\text {eff }}>5000 \mathrm{~K}$. The triangles in the diagram represent the disks that we detected; small squares represent upper limits. The distribution of detections and upper limits makes clear that we detected all disks with $\tau>210^{-5}$ or $M_{\mathrm{d}} 1.010^{-5} M_{\oplus}$ around the $A, F, G$-type stars in our sample of 84 stars; we may, however, have missed a few disks around our $\mathrm{K}$ stars and we may have missed truncated and thus hot disks.

\subsection{The incidence and survival of remnant dust disks}

The results discussed here have also been presented in Habing et al. (1999).

Stellar ages have been derived in an accompanying paper (Lachaume et al. 1999). Errors in the determination of the ages have been given in that paper; occasionally they may be as large as a factor of 2 to 3 ; errors that large will not detract from our main conclusions.
Table 7. Average distances of stars with and without a disk

\begin{tabular}{|c|cc|cc|}
\hline & $\#$ & $\begin{array}{c}\text { without disk } \\
(\mathrm{pc})\end{array}$ & $\#$ & $\begin{array}{c}\text { with disk } \\
(\mathrm{pc})\end{array}$ \\
\hline $\mathrm{A}^{* *}$ & 9 & $22.6 \pm 11.0$ & 6 & $16.8 \pm 7.1$ \\
$\mathrm{~F}^{* *}$ & 21 & $14.6 \pm 4.6$ & 2 & $14.0 \pm \ldots$ \\
$\mathrm{G}^{* *}$ & 17 & $12.3 \pm 3.9$ & 4 & $11.3 \pm 5.3$ \\
$\mathrm{~K}^{* *}$ & 20 & $9.5 \pm 3.8$ & 2 & $6.6 \pm \ldots$ \\
\hline
\end{tabular}

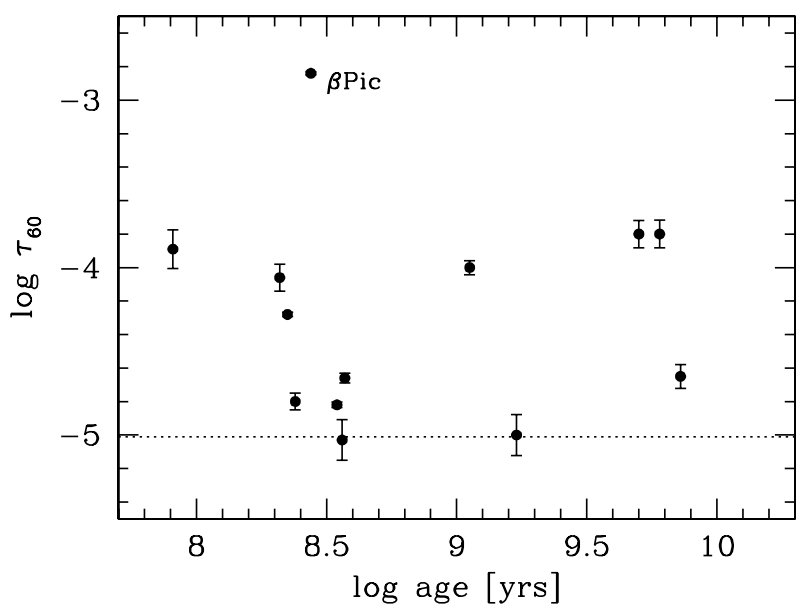

Fig. 6. $\tau$, the fraction of the stellar light reemitted at infrared wavelengths, is shown as a function of stellar age

Table 8. Detection statistics

\begin{tabular}{|c|cc|cc|cc|cc|}
\hline & \multicolumn{2}{|c|}{$<400$} & \multicolumn{2}{c|}{$400-1000$} & \multicolumn{2}{c|}{$1.0-5.0$} & \multicolumn{2}{c|}{$>5.00$} \\
& Myr & \multicolumn{2}{c|}{ Myr } & \multicolumn{2}{c|}{ Gyr } & \multicolumn{2}{c|}{ Gyr } \\
& tot & disk & tot & disk & tot & disk & tot & disk \\
\hline $\mathrm{A}^{* *}$ & 10 & 6 & 4 & 0 & 1 & 0 & 0 & 0 \\
$\mathrm{~F}^{* *}$ & 0 & 0 & 1 & 0 & 17 & 2 & 5 & 0 \\
$\mathrm{G}^{* *}$ & 2 & 1 & 0 & 0 & 7 & 0 & 12 & 3 \\
$\mathrm{~K}^{* *}$ & 3 & 2 & 2 & 0 & 5 & 0 & 12 & 0 \\
& & & & & & & & \\
total & 15 & 9 & 7 & 0 & 30 & 2 & 29 & 3 \\
\hline
\end{tabular}

\subsubsection{The question of completeness and statistical bias}

Our sample has been selected from the catalogue of stars within 25 pc from the Sun by Woolley et al. (1970); this catalogue is definitely incomplete and so must be our sample. Even within the distance limits given in Table 1 stars will exist that we could have included but did not. This incompleteness does not, however, introduce a statistical bias: we have checked that for a given spectral type the distribution of the stellar distances is the same for stars with a disk as for stars without a disk; this is illustrated by the average distances in Table 7 .

\subsubsection{Detection statistics and stellar age}

Figure 6 presents graphically the fraction of the (visual) stellar light reemitted in the infrared by the disk as a function of the stellar age. Similar diagrams based mainly on IRAS results, have been published before- see, for 
example, Holland et al. (1998). A general, continuous correlation appears: disks around PMS-stars (e.g. Herbig $\mathrm{AeBe})$ are more massive than disks around stars like $\beta$ Pic and Vega, and the disk around the Sun is still less massive. These earlier diagrams have almost no data on the age range shown in Fig. 6 and the new ISO data fill in an important hole.

Table 8 summarizes the detections at $60 \mu \mathrm{m}$ separately for stars of different age and of different spectral type together with the same numbers for stars with a disk; in the column marked "tot" the total number of stars (disks plus no-disks) is shown and under the heading "disk" the number of stars with a disk. The total count is 81 instead of 84 because for three of our target stars (two A-stars and one K-star) the age could not be estimated in a satisfying manner. Table 8 shows that the stars with a detected disk are systematically younger than the stars without disk: out of the 15 stars younger than 400 Myr nine (60\%) have a disk; out of the 66 older stars only five have a disk (8\%). Furthermore, there exists a more or less sharply defined age above which a star has no longer a disk. This is best demonstrated by the A-stars. Six A-stars have a disk; the stellar ages are 220, 240, 280, 350, 360, 380 Myr. For the A-stars without disk the corresponding ages are 300,320 , 350, 380, 420, 480, 540, 890, 1230 Myr: 350 to $400 \mathrm{Myr}$ is a well-defined transition region. We conclude that the $\mathrm{A}$ stars in general arrive on the main-sequence with a disk, but that they loose the disk within $50 \mathrm{Myr}$ when they are about 350 Myr old.

Is what is true for the A-stars also valid for the stars of other spectral types? Our answer is "probably yes": of the five F, G, and K stars younger than $400 \mathrm{Myr}$ three $(60 \%)$ have a disk. Of the $61 \mathrm{~F}, \mathrm{G}$, and $\mathrm{K}$ stars older than 400 Myr five have a disk (one in twelve or $8 \%$ ). The percentages are the same as for the A-stars but the $60 \%$ for young G- and K-stars is based on only three detections. It seems that the disks around $\mathrm{F}, \mathrm{G}$, and $\mathrm{K}$ stars decay in a similarly short time after arrival on the main sequence.

An immediate question is: do all stars arrive at the main sequence with a disk? Studies of pre-main-sequence stars show that disks are common, but whether they always exist is unknown. The sequence of ages of the A-stars shows that the three youngest A-stars have a disk. This suggests that all stars arrive on the main sequence with a disk, but the suggestion is based on small-number statistics. We therefore leave the question without an answer but add two relevant remarks without further comment: some very young stars have no detectable disk, for example HD 116842 (A5V, 320 Myr), HD 20630 (G5V, 300 Myr), HD 37394 (K1V, $320 \mathrm{Myr}$ ) and some old stars have retained their disk; examples: HD 10700 (G8V, 7.2 Gyr), HD 75732 (G5V, 5.0 Gyr) and HD 207129 (G0V, 6.0 Gyr); the last case has been studied in detail (Jourdain de Muizon et al. 1999).

The age effect is shown graphically in Fig. 7; it displays the cumulative distribution of stars with a disk. The $x$-axis is the index of a star after all stars have been sorted by age. At a given age the local slope of the curve in this diagram

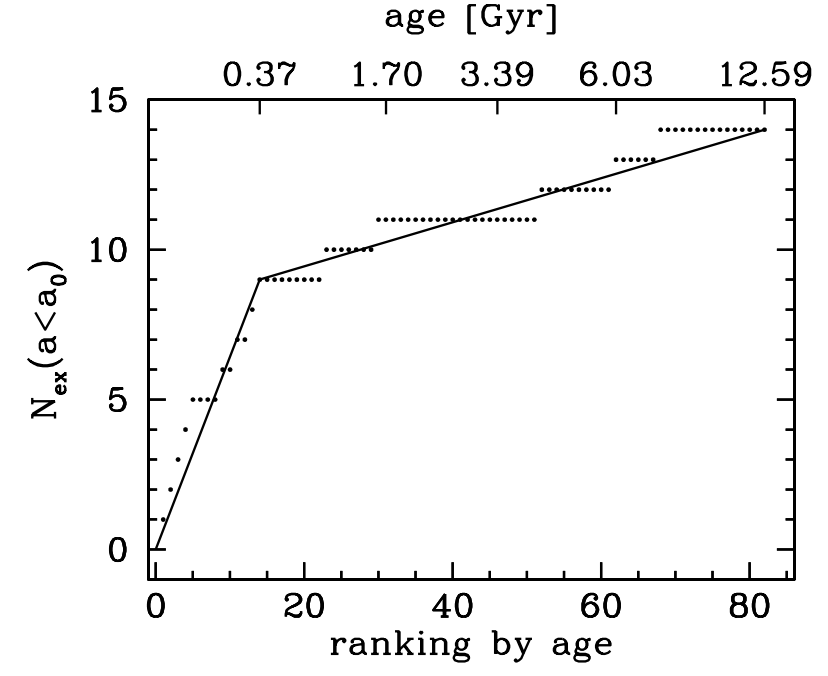

Fig. 7. Cumulative distribution of excess stars, as a function of the index after sorting by age. The two segments of a continuous straight line are predicted by assuming that in the first $400 \mathrm{Myr}$ the rate of disappearance of disks is much higher than afterwards (see text)

gives the probability that stars of that age have a disk. The two line segments shows how the cumulative number increases when $70 \%$ of the disks disappear gradually in the first $0.4 \mathrm{Gyr}$ and the remaining $30 \%$ gradually in the 12 Gyr thereafter.

In Sect. 5.6 we will review the evidence that at about 400 Myr after the formation of the Sun a related phenomenon took place in the solar system.

\subsection{The need to continuously replenish the dust particles}

In "Vega-like" circumstellar disks the dust particles have a life-time much shorter than the age of the star. Within 1 Myr they will disappear via radiation pressure and the Poynting-Robertson effect (Aumann et al. 1984). An upper limit of $10^{6}$ year is given for dust around A-type stars by Poynting-Robertson drag (Burns et al. 1979; Backman \& Paresce 1993); the actual life time will be smaller: for $\beta$ Pic, Artymowicz \& Clampin (1997) find only 4000 years. Continuously new grains have to replace those that disappear. A plausible mechanism that can supply these new grains at a high enough rate and for a sufficient long time are the collisions between asteroids and planetesimals. Direct detection of such larger bodies is not yet possible although the existence of comets around $\beta$ Pic is suggested by the rapidly appearing and disappearing components of the CaII K-absorption line (Ferlet et al. 1987). The total mass of the dust that ISO detected is about that of the Moon. To produce the dust for $400 \mathrm{Myr}$ much more mass must be present in invisible form, that of asteroids or "planetesimals". Thus the disappearance of the infrared excess on a timescale of 400 Myr does not trace the removal of the dust grains, but the lifetime of 
Table 9. Multiple systems with a dust disk

\begin{tabular}{|rrr|rrr|}
\hline Name & HD & CCDM & $N_{\text {tot }}$ & $r$ & $\Delta m$ \\
& & & & $\mathrm{AU}$ & \\
\hline$\tau$ Cet & 10700 & $01441-1557$ & 1 & 328 & 9.5 \\
$\rho^{1}$ Cnc & 75732 & $08526+2820$ & 1 & 1100 & 7.2 \\
$\beta$ Leo & 102647 & $11490+1433$ & 3 & 440 & 13.5 \\
$\sigma$ Boo & 128167 & $14347+2945$ & 2 & 3700 & 5.3 \\
$\alpha$ Lyr & 172167 & $18369+3847$ & 4 & 490 & 9.4 \\
& 207129 & $21483-4718$ & 1 & 860 & 3.0 \\
\hline
\end{tabular}

the disk or planetesimals that replenishes the dust. In the solar system the same may have happened; see below.

\subsection{Disks in the presence of a companion star or a planet}

When a star has a companion or a planet the gravitational field will have a time-variable component. Will this component destroy the disk? Not necessarily so: the planets Jupiter and Saturn have both a dust disk and many satellites.

On purpose we did not select narrow binaries: we rejected stars within 1 arcmin from a target star, unless this other star was at least 5 magnitudes fainter in the $V$-band. This criterium accepts wide multiple-stars and indeed these occur. We used the Hipparcos Catalogue to check all 84 stars from Table 2 for multiplicity. Forty-eight stars have an entry in the "Catalogue of companions of double and multiple stars" (= CCDM), see Dommanget \& Nys (1994). Among the 14 stars with a disk there are seven wide multiple-stars. In one case (HD 22049) the star is part of an astrometric double; we ignore the object. That leaves us with six stars that have both a disk and stellar companions. The conclusion is therefore that companions do not necessarily destroy a disk.

Table 9 contains information on these six stars with both a disk and (at least) one companion. In Col. (1) the name appears, in Col. (2) the HD-number and in Col. (3) the entry-number in the CCDM; Col. (4) gives the total number of companions given in the CCDM, Col. (5) gives the distance, $r$, between $\mathrm{A}$ and $\mathrm{B}$ in astronomical units and Col. (6) the magnitude difference in the $V$-band between the first and the second component ("A" and "B", respectively).

There are at least two remarkable cases in Table 9. One is Vega (HD 172167) with four companions; its brightest companion is at $490 \mathrm{AU}$, but its closest companion at only $200 \mathrm{AU}$, just outside of Vega's disk. The other is $\rho^{1} \mathrm{Cnc}$ that has a disk (Dominik et al. 1998; Trilling \& Brown 1998; Jayawardhana et al. 2000), a planet (Butler et al. 1997) and a stellar companion.

The data in Table 9 thus show that disks are found in wide multiple-systems: multiplicity does not necessarily destroy a disk.

\subsection{The connection to the solar system}

The solar system shows evidence for a fast removal of a disk of planetesimals a few hundred Myr after the Sun formed a disk. The best case is given by the surface of the Moon, where accurate crater counting from high resolution imaging can be combined with accurate age determinations of different parts of the Moon's surface. The age of the lunar surface is known from the rocks brought back to earth by the Apollo missions; the early history of the Moon was marked by a much higher cratering rate than observed today; see for a discussion Shoemaker \& Shoemaker (1999). This so-called "heavy bombardment" lasted until some $600 \mathrm{Myr}$ after the formation of the Sun. Thereafter the impact rate decreased exponentially with time constants between $10^{7}$ and a few times $10^{8}$ years (Chyba 1990).

Other planets and satellites with little erosion on their surface confirm this evidence: Mercury (Strom \& Neukum 1988), Mars (Ash et al. 1996; Soderblom et al. 1974) Ganymede and Callisto (Shoemaker \& Wolfe 1982; Neukum et al. 1997; Zahnle et al. 1998). The exact timescales are a matter of debate. Thus there are indications of a cleanup phase of a few hundred Myr throughout the solar system; these cleanup processes may be dynamically connected.

\section{Conclusions on the incidence of remnant disks}

The photometers on ISO have been used to measure the 60 and $170 \mu \mathrm{m}$ flux densities of a sample of 84 main-sequence stars with spectral types from A to K.

On the basis of the evidence presented we draw the following conclusions:

- Fourteen stars have a flux in excess of the expected photospheric flux. We conclude that each of these has a circumstellar disk that is a remnant from its premain-sequence time. Two more stars may have a disk, but there is a significant chance that the emission is due to a background galaxy;

- The overall incidence of disks is $14 / 84$ or $17 \%$. A-stars have a higher incidence than the other stars;

- We prove that the detectability of a given disk is the same for A, F and G-stars with the same photospheric flux at $60 \mu \mathrm{m} ; \mathrm{K}$ stars have a lower probability of detection;

- The disks that we detect have a value of $\tau$, between $210^{-4}$ and $410^{-6}$. The upper limit is real: mainsequence stars do not carry stronger disks; the exception is $\beta$ Pic with $\tau=810^{-3}$. The lower limit is caused by selection effects: fainter disks are below our detection threshold;

- Six out of the ten A-type stars younger than $400 \mathrm{Myr}$ have a disk; the disk is absent around all five older A-type stars: the disks disappear around this age;

- Disks around F-, G- and K-stars probably disappear on a similar time scale. The disappearance of disks is 
not a continuous process; $400 \mathrm{Myr}$ is the age at which most disks disappear;

- In the history of our solar system the abrupt ending of the initial "heavy bombardment" of the Moon has the same time scale;

- We suggest that the disks that we detect are actually sites where a "heavy bombardment" takes place now. The time scale on which the disks disappear is actually the time scale of the disappearance of the bombarding planetesimals;

- The mass that we detect through its infrared emission is only a minute fraction (about $10^{-5}$ ) of the mass present. We see the gravel but not the very big stones that produce it;

- Some very young stars lack a disk; some very old stars still have a disk: the existence of both groups needs to be explained;

- Stars in multiple systems retain their remnant disks as often as isolated stars.

Acknowledgements. The ISOPHOT data presented in this paper were reduced using PIA, which is a joint development by the ESA Astrophysics Division and the ISOPHOT consortium. In particular, we would like to thank Carlos Gabriel for his help with PIA. We also thank J. Dommanget for helpful information on the multiplicity of our stars and the referee, R. Liseau for his careful comments. This research has made use of the Simbad database, operated at CDS, Strasbourg, France, and of NASA's Astrophysics Data System Abstract Service. CD was supported by the Stichting Astronomisch Onderzoek in Nederland, Astron project 781-76-015.

\section{Appendix A: Observing strategy for minimaps}

The observations at $60 \mu \mathrm{m}$ and $170 \mu \mathrm{m}$ have been taken as minimaps with the $\mathrm{C} 100$ and C200 detector arrays using $3 \times 3$ rastersteps; see Fig. A.1. In this figure the upper half shows the labeling, " $p$ ", of the 9 , respectively 4 pixels (detectors) for the C100 and C200 arrays. The lower diagram gives the numbering, " $r$ ", of the successive array positions as it moves over the sky; the raster step is 46 arcsec for both arrays. Consider first a measurement with the $\mathrm{C} 100$ array. At raster step $r=1$ the source illuminates pixel $p=7$; at the next step, $r=2$, the source illuminates $p=4$, at $r=3$ the source is on $p=1$, etc. For the C200 measurements $r=1$ has the source on $p=1$; at $r=2$ the source is half on $p=1$ and half on $p=2$; at $r=3$ the source is on $p=2$, etc.

\section{Appendix B: Data reduction}

We used the following procedure to extract the flux. The result of a minimap measurement is a flux per pixel for each pixel and each raster position. Let $f(p, r)$ be the measured flux in pixel $p$ at raster position $r$. There are $n_{p}$ pixels and $n_{r}$ raster positions. We first calculate a flat field correction $f_{\text {flat }}(p)$ for each pixel by assuming that at
C100

\begin{tabular}{|l|l|l|}
\hline 9 & 6 & 3 \\
\hline 8 & 5 & 2 \\
\hline 7 & 4 & 1 \\
\hline
\end{tabular}

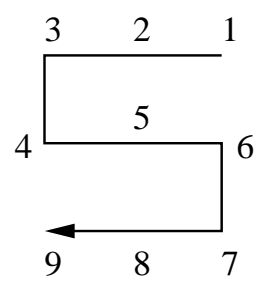

C200
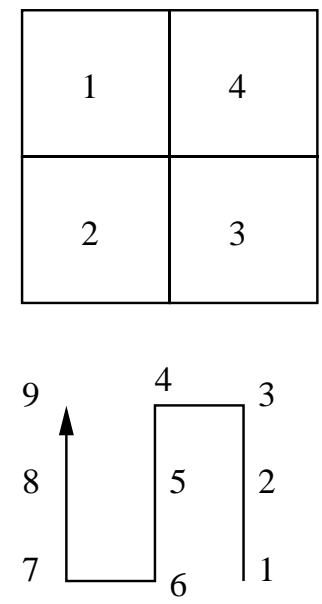

Fig. A.1. The upper half labels the different pixels of the $\mathrm{C} 100$ and C200 detectors seen in projection on the sky. The lower half describes the stepping directions: see text

Table B.1. Weight factors for minimap flux determination

\begin{tabular}{|c|rrrrrrrrr|}
\hline & \multicolumn{10}{|c|}{ Raster position } \\
Pix & 1 & 2 & 3 & 4 & 5 & 6 & 7 & 8 & 9 \\
\hline C100 & & & & & & & & & \\
1 & 0 & 0 & 1 & 0 & 0 & $\left(-\frac{1}{3}\right)$ & $-\frac{1}{3}$ & $-\frac{1}{3}$ & 0 \\
2 & $-\frac{1}{3}$ & 0 & 0 & 1 & 0 & $\left(-\frac{1}{3}\right)$ & $-\frac{1}{3}$ & 0 & 0 \\
3 & $-\frac{1}{3}$ & $-\frac{1}{3}$ & 0 & 0 & 0 & $\left(-\frac{1}{3}\right)$ & 0 & 0 & 1 \\
4 & 0 & 1 & 0 & 0 & 0 & $(0)$ & $-\frac{1}{3}$ & $-\frac{1}{3}$ & $-\frac{1}{3}$ \\
5 & $-\frac{1}{4}$ & 0 & $-\frac{1}{4}$ & 0 & 1 & $(0)$ & $-\frac{1}{4}$ & 0 & $-\frac{1}{4}$ \\
6 & $-\frac{1}{3}$ & $-\frac{1}{3}$ & $-\frac{1}{3}$ & 0 & 0 & $(0)$ & 0 & 1 & 0 \\
7 & 1 & 0 & 0 & $-\frac{1}{3}$ & 0 & $(0)$ & 0 & $-\frac{1}{3}$ & $-\frac{1}{3}$ \\
8 & 0 & 0 & $-\frac{1}{3}$ & $-\frac{1}{3}$ & 0 & $(1)$ & 0 & 0 & $-\frac{1}{3}$ \\
9 & 0 & $-\frac{1}{3}$ & $-\frac{1}{3}$ & $-\frac{1}{3}$ & 0 & $(0)$ & 1 & 0 & 0 \\
\hline C200 & & & & & & & & & \\
1 & 1 & 0 & 0 & 0 & 0 & 0 & 0 & 0 & -1 \\
2 & 0 & 0 & 1 & 0 & 0 & 0 & -1 & 0 & 0 \\
3 & -1 & 0 & 0 & 0 & 0 & 0 & 0 & 0 & 1 \\
4 & 0 & 0 & -1 & 0 & 0 & 0 & 1 & 0 & 0 \\
\hline
\end{tabular}

one raster position the flux averaged over all pixels is the same

$f_{\text {flat }}(p)=\frac{\frac{1}{n_{p}} \sum_{p^{\prime}=1}^{n_{p}} \sum_{r^{\prime}=1}^{n_{r}} f\left(p^{\prime}, r^{\prime}\right)}{\sum_{r^{\prime}=1}^{n_{r}} f\left(p, r^{\prime}\right)}$.

Since the individual pixels in the C100/C200 cameras have different properties, we use the measurements of each pixel to derive a separate measurement of the source flux. In order to compute the background-subtracted source flux, we assign to each raster position a weight factor $g(p, r)$. Since the exact point spread function is not known 
well enough, we use as on-source measurement the raster position where the pixel was centered on the source (weight factor 1). The background measurement is derived by averaging over the raster postions where the same pixel $p$ was far away from the source (weight $-1 / 3$ or $-1 / 4$ ). Raster positions in which the pixel was partially on the source are ignored (weight 0 ). The resulting weight factors are given in Table B.1. The source flux measured by pixel $p$ is given by

$F(p)=\frac{f_{\text {flat }}}{f_{\mathrm{psf}}} \sum_{r^{\prime}=1}^{n_{r}} g\left(p, r^{\prime}\right) f\left(p, r^{\prime}\right)$

$f_{\mathrm{psf}}$ is the point spread function correction factor as given by Laureijs et al. (2000). We then derive the flux $F$ and the error $\sigma$ by treating the different $F(p)$ as independent measurements.

$F=\frac{1}{n_{p}} F(p)$

$\sigma=\frac{1}{\sqrt{n_{p}-1}} \sqrt{\sum_{p^{\prime}=1}^{n_{p}}\left(F\left(p^{\prime}\right)-F\right)^{2}}$.

The point spread function is broader than a pixel. We have corrected for this, using the parameter $f_{\text {psf }}$ given above. The correction factor may be too low: Dent et al. (2000) show that the disk around Fomalhaut ( $\alpha$ PsA) is extended compared to our point-spread function. This means that all our $60 \mu \mathrm{m}$ and $170 \mu \mathrm{m}$ detections are probably somewhat underestimated.

We have ignored pixel 6 of the C100 camera entirely, because its characteristics differ significantly from those of the other pixels: it has a much higher dark signal and anomalous transient behaviour.

In the future the characteristics of each pixel will be determined with increasing accuracy. It may prove worthwhile to redetermine the fluxes again.

\section{Appendix C: Optical depth of the disk, detection limit and illumination bias}

We discuss how the contrast factor, $C_{60}$, depends on the spectral type of the star, $T_{\text {eff }}$, and on the optical depth, $\tau$, of the disk. The dust grains in the remnant disk are relatively large, at least in cases where a determination of the grain size has been possible (Bliek et al. 1994; Artymowicz et al. 1989) and the absorption efficiency for stellar radiation will be high for stars of all spectral types. The efficiency for emission is low: the dust particles emit beyond $30 \mu \mathrm{m}$ and these wavelengths are larger than that of the particles. We assume that the dust grains are all of a single size, $a$, and located at a single distance, $r$, from the star. We will introduce various constants that we will call $A_{i}, i=0-6$.

$C_{60} \equiv \frac{L_{\nu, \mathrm{d}}}{L_{\nu, *}}=\frac{L_{\nu, \mathrm{d}}}{L_{d}} \cdot \frac{L_{\mathrm{d}}}{L_{*}} \cdot \frac{L_{*}}{L_{\nu, *}}$.
First we determine $L_{\nu, \mathrm{d}}$, the luminosity of the disk at the frequency $\nu$, and $L_{\mathrm{d}}$, the total luminosity of the disk:

$L_{\nu, \mathrm{d}}=N 4 \pi^{2} a^{2} Q_{\nu} B_{\nu}\left(T_{\mathrm{d}}\right)$

and

$L_{\mathrm{d}}=N 4 \pi^{2} a^{2} \int_{0}^{\infty} Q_{\nu} B_{\nu}\left(T_{\mathrm{d}}\right) \mathrm{d} \nu$.

We consider dust emission at $60 \mu \mathrm{m} ; B_{\nu}(T)$ can be approximated by the Wien-equation. We thus write:

$L_{\nu, \mathrm{d}}=A_{0} \exp \left(-\frac{240 \mathrm{~K}}{T_{\mathrm{d}}}\right)$

Define the average absorption efficiency:

$Q_{\text {ave }}\left(T_{\mathrm{d}}\right) \equiv \pi \int_{0}^{\infty} Q_{\nu} B_{\nu}\left(T_{\mathrm{d}}\right) \mathrm{d} \nu /\left(\sigma T_{\mathrm{d}}^{4}\right)$

For low dust-temperatures $Q_{\text {ave }}$ can be approximated by $Q_{\text {ave }}=A_{1} T_{\mathrm{d}}^{\alpha}$ with $\alpha \approx 2$ (Natta \& Panagia 1976) and thus

$L_{\mathrm{d}}=A_{2} T_{\mathrm{d}}^{6}$.

Second, we determine the stellar luminosity, $L_{\nu, *}$, at frequency $\nu$ and the total stellar luminosity, $L_{*}$, both by ignoring the effects of dust, that is the luminosity at the photospheric level. The photospheric emission is approximated by the Rayleigh-Jeans equation:

$L_{\nu, *}=\frac{\pi B_{\nu}\left(T_{\mathrm{eff}}\right)}{\sigma T_{\mathrm{eff}}^{4}} L_{*}=\frac{A_{3} L_{*}}{T_{\mathrm{eff}}^{3}}$.

The stellar luminosity for main sequence stars of spectral type A0-K5 can be approximated within $30 \%$ by:

$L_{*}=A_{4} T_{\mathrm{eff}}^{8.2}$.

Third, we determine the relation between $T_{\mathrm{d}}$ and $T_{\text {eff }}$. For photospheric temperatures $Q_{\text {ave }}$ is independent of $T_{\text {eff }}$. The energy absorbed by a grain is thus $\propto L_{*} \propto T_{\text {eff }}^{8.2}$. The energy emitted is $\propto T_{\mathrm{d}}^{6}$. Because the energy emitted equals the energy absorbed we conclude that $T_{\text {eff }}=A_{5} T_{\mathrm{d}}^{6 / 8.2}$. Combining these results we find

$C_{60}=A_{6} \cdot \frac{1}{T_{\mathrm{d}}^{3.8}} \cdot \exp \left(-\frac{240}{T_{\mathrm{d}}}\right)$.

We have calculated $C_{60}$ without making the various approximations in Eqs. (C.1) through (C.7): Fig. C.1 shows the results. The results are valid if the distance dustring/star is the same (50 AU) for all stars irrespective of the spectral type.

In the figure we assume that for an A0-star $C_{60}$ has the value 1 and $T_{\mathrm{d}}=80$ or $120 \mathrm{~K}$. For a star of later spectral type, the dust will be cooler and will emit less energy (see Eq. (C.6)), but since $\lambda=60 \mu \mathrm{m}$ is at the Wien-side of the black body curve, the emission at $60 \mu \mathrm{m}$ will increase- see Eq. (C.4). The consequence is that $C_{60}$ remains constant for A-, F- and early G-type stars. For late G- and for K- and M-type stars the dust becomes too cold to be detected at $60 \mu \mathrm{m}$. Only photometry at longer wavelengths will ultimately be able to detect such very cold disks. 


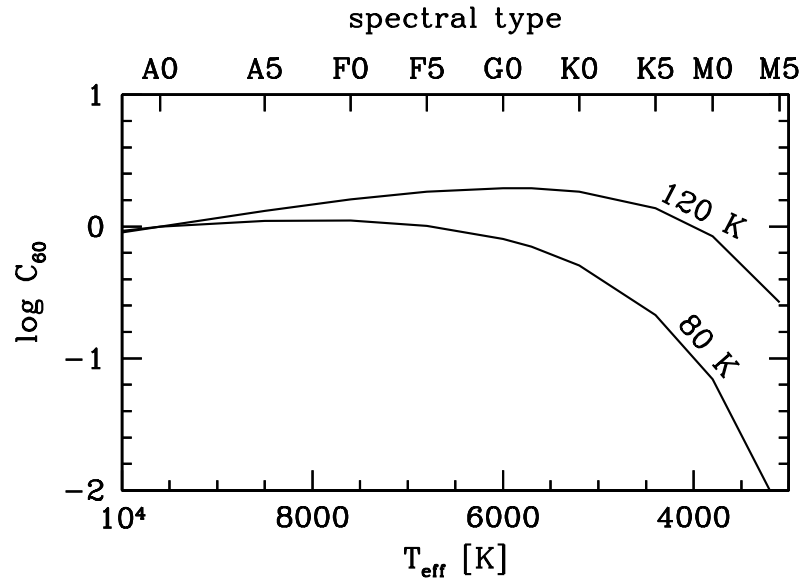

Fig. C.1. The contrast factor $C_{60} \equiv F_{\nu}^{\mathrm{d}} / F_{\nu}^{\text {pred }}$ for a ring of dust at a distance of $50 \mathrm{AU}$ from the star and for stars of different effective temperature. We assumed $T_{\mathrm{d}}$ to be 80 or $120 \mathrm{~K}$ for an A0 star

\section{Appendix D: Determination of $\tau$ from observed fluxes}

For most of our stars we have only a detection of the disk at $60 \mu \mathrm{m}$. To calculate the optical depth and the mass of the disk we need an estimate of the disk emission integrated over all wavelengths. If the dust around an A star has a temperature of $\stackrel{\circ}{\mathrm{d}}_{\mathrm{d}}$, the stars of later types will have lower dust temperatures. Numerical evaluation shows that, assuming constant distance between the star and the dust, the following relation is a good approximation

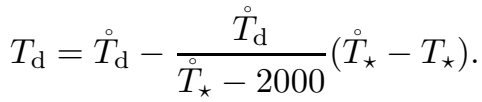

With this estimate of the dust temperature, a single flux determination is sufficient to determine the the fractional luminosity. We have used this method to determine estimates of $\tau$ independently from all wavelength where we have determined an excess. We used $\stackrel{\circ}{\star}_{\star}=9600 \mathrm{~K}$ and $\stackrel{\circ}{\mathrm{T}}_{\mathrm{d}}=80 \mathrm{~K}$, values that agree with those measured for Vega.

\section{References}

Ábrahám, P., Leinert, C., Burkert, A., et al. 1998, A\&A, 338, 91

Artymowicz, P., Burrows, C., \& Paresce, F. 1989, ApJ, 337, 494

Artymowicz, P., \& Clampin, M. 1997, ApJ, 490, 863

Ash, R. D., Knott, S. F., \& Turner, G. 1996, Nat, 380, 57

Aumann, H. 1985, PASP, 97, 885

Aumann, H., Gillett, F., Beichman, C., et al. 1984, ApJ, 278, L23

Backman, D., \& Paresce, F. 1993, in Protostars and Planets III, ed. E. Levy, \& J. Lunine (University of Arizona, Tucson), 208-2000

Beckwith, S., \& Sargent, A. 1996, Nat, 383

Bliek, N. v. d., Prusti, T., \& Waters, L. 1994, A\&A, 285

Burns, J. A., Lamy, P. L., \& Soter, S. 1979, Icarus, 40, 1

Butler, R., Marcy, G. W., et al. 1997, ApJL, 474, L115

Chini, R., Kruegel, E., Kreysa, E., et al. 1991, A\&A, 252, 220
Chyba, C. F. 1990, Nat, 343, 129

Dent, W., Walker, H., Holland, W., et al. 2000, MNRAS, 314, 702

Dole, H., Gispert, R., Lagache, G., et al. 2000, in ISO Surveys of a Dusty Universe, ed. D. Lemke, M. Stickel, \& K. Wilke (Springer Verlag) [astro-ph/0002283]

Dominik, C., Laureijs, R., Jourdain de Muizon, M., et al. 1998, A\&A, 329, L53

Dommanget, J., \& Nys, O. 1994, Comm. Obs. R. Belgique, Ser. A., 115

Draine, B., \& Lee, H. 1984, ApJ, 285, 89

Elbaz, D., Cesarsky, C., Fadda, D., et al. 2000, A\&A Lett, in press, [astro-ph/9910406]

Ferlet, R., Hobbs, L., \& Vidal-Madjar, A. 1987, A\&A, 185, 267

Gillett, F. 1986, in Light on dark matter, ed. F. Israel (Reidel, Dordrecht), 61

Habing, H., Bouchet, P., Dominik, C., et al. 1996, A\&A, 315, L233

Habing, H., Dominik, C., Jourdain de Muizon, M., et al. 1999, Nat, 401, 456

Hobbs, L., Vidal-Madjar, A., Ferlet, R., et al. 1985, ApJL, 293, L29

Holland, W., Greaves, J., Zuckerman, B., et al. 1998, Nat, 392, 788

Jayawardhana, R., Holland, W., Greaves, J., et al. 2000, ApJ, 536,425

Johnson, H., \& Wright, C. 1983, ApJS, 53, 643

Jourdain de Muizon, M., Laureijs, R., Dominik, C., et al. 1999, A\&A, 350, 875

Kamp, I., \& Bertoldi, F. 2000, A\&A, 353, 276

Kessler, M., Steinz, J., Anderegg, M., et al. 1996, A\&A, 315, L27

Klaas, U., Krüger, H., Heinrichsen, I., et al. 1994, ISOPHOT Observer's Manual, Version 3.1 (ESA Publication)

Kunzli, M., North, P., Kurucz, R. L., et al. 1997, A\&AS, 122, 51

Lachaume, R., Dominik, C., Lanz, T., et al. 1999, A\&A, 348, 897

Laureijs, R., Klaas, U., Richards, P., et al. 2000, ISO Handbook, vol. V: PHT-The Imaging Photo-Polarimeter version 1.0, ISO Data Centre,

(url: www.iso.vilspa.esa.es/manuals/HANDBOOK/V/)

Lemke, D., Klaas, U., Abolins, J., et al. 1996, A\&A, 315, L64

Liseau, R. 1999, A\&A, 348, 133

Mannings, V., \& Barlow, M. 1998, ApJ, 497, 330

Matsuhara, H., Kawara, K., Sato, Y., et al. 2000, A\&A, accepted, [astro-ph/0006444]

Natta, A., \& Panagia, N. 1976, A\&A, 50, 191

Neukum, G., Wagner, R., Wolf, U., et al. 1997, BAAS, 29, 984

Oliver, S., Serjeant, S., Efstathiou, A., et al. 2000, in ISO Surveys of a Dusty Universe, ed. D. Lemke, M. Stickel, \& K. Wilke (Springer Verlag), [astro-ph/0005279]

Perryman, M., O'Flaherty, K., van Leeuwen, F., et al. 1997, The Hipparcos and Tycho Catalogues, ESA SP-1200

Plets, H., \& Vynckier, C. 1999, A\&A, 343

Plets, H., Waelkens, C., Oudmaijer, R., et al. 1997, A\&A, 323, 513

Sargent, A., \& Welch, W. 1993, ARA\&A., 31, 297

Shoemaker, E., \& Shoemaker, C. 1999, in The new solar system, 4th edition, ed. J. K. Beatty, C. Petersen, \& A. Chaikin (Sky Publishing Corporation and Cambridge University Press), 69

Shoemaker, E. M., \& Wolfe, R. A. 1982, in Satellites of Jupiter, ed. D. Morrison (Tucson: Univ. of Arizona Press), 277 
Smith, B., \& Terrile, R. 1984, Sci, 226, 1421

Soderblom, L. A., Condit, C. D., West, R. A., et al. 1974, Icarus, 22, 239

Strom, R. G., \& Neukum, G. 1988, in Mercury (University of Arizona Press), 336

Trilling, D., \& Brown, R. 1998, Nat, 395, 775

van Dishoeck, E., \& Blake, G. A. 1998, ARA\&A, 317
Walker, H., \& Wolstencroft, R. 1988, PASP, 100, 1509

Waters, L., Coté, J., \& Aumann, H. 1987, A\&A, 172, 225

Weissman, P. R. 1984, Sci, 224, 987

Woolley, R., Epps, E., \& Penston, M., et al. 1970, Royal Obs. Ann., 5

Zahnle, K., Dones, L., \& Levison, H. F. 1998, Icarus, 136, 202

Zuckerman, B., \& Becklin, E. E. 1993, APJ, 414, 793 